\title{
On the Relationship between ENSO, Stratospheric Sudden Warmings, and Blocking
}

\author{
DAVID BARRIOPEDRO
}

\begin{abstract}
Departamento de Física de la Tierra II, Facultad de Ciencias Físicas, Universidad Complutense de Madrid, and Instituto de Geociencias, Centro Mixto del Consejo Superior de Investigaciones Científicas-Universidad Complutense de Madrid, Madrid, Spain
\end{abstract}

\author{
NATALia CALVO
}

Departamento de Física de la Tierra II, Facultad de Ciencias Físicas, Universidad Complutense de Madrid, Madrid, Spain

(Manuscript received 10 December 2013, in final form 8 March 2014)

\begin{abstract}
This paper examines the influence of El Niño-Southern Oscillation (ENSO) on different aspects of major stratospheric sudden warmings (SSWs), focusing on the precursor role of blocking events. The results reveal an ENSO modulation of the blocking precursors of SSWs. European and Atlantic blocks tend to precede SSWs during El Niño (EN), whereas eastern Pacific and Siberian blocks are the preferred precursors of SSWs during La Niña (LN) winters. This ENSO preference for different blocking precursors seems to occur through an ENSO effect on regional blocking persistence, which in turn favors the occurrence of SSWs. The regional blocking precursors of SSWs during each ENSO phase also have different impacts on the upward propagation of planetary-scale wavenumbers 1 and 2; hence, they determine ENSO differences in the wavenumber signatures of SSWs. SSWs occurring during EN are preceded by amplification of wavenumber 1, whereas LN SSWs are predominantly associated to wavenumber-2 amplification. However, there is not a strong preference for splitting or displacement SSWs during any ENSO phase. This is mainly because during EN, splitting SSWs do not show a wavenumber-2 pattern.
\end{abstract}

\section{Introduction}

Major stratospheric sudden warmings (SSWs) are foremost disruptions of the stratospheric polar vortex associated with enhanced upward propagation of planetary Rossby waves from the troposphere into the stratosphere during winter (Matsuno 1971; Andrews et al. 1987). SSWs are more frequent in the Northern Hemisphere $(\mathrm{NH})$, and exhibit large interdecadal variability (e.g., Gómez-Escolar et al. 2012). During SSWs the polar vortex is either displaced equatorward (displacement type) or broken into two parts (splitting type) (e.g., Andrews et al. 1987). This is accompanied by a reversal of the westerly winds over the polar cap and a dramatic warming of the polar stratosphere (e.g., Limpasuvan et al.

Corresponding author address: David Barriopedro, Departamento de Física de la Tierra II, Facultad de Ciencias Físicas, Universidad Complutense de Madrid (UCM/IGEO), Avenida Complutense, s/n, Ciudad Universitaria, 28040 Madrid, Spain.

E-mail: dbarriop@fis.ucm.es
2004). Subsequently, these stratospheric anomalies in the zonal mean flow progress downward within a few weeks into the troposphere, where they persist from weeks to months (e.g., Baldwin and Dunkerton 2001). In recent years, several studies have stressed the importance of the stratospheric anomalies associated with SSWs to provide more skilful extended-range and seasonal forecasts of tropospheric winter weather in the northern extratropical regions (e.g., Baldwin et al. 2003). In this sense, the classification of SSWs into splitting and displacement events is important since the tropospheric responses differ according with the type of SSW (Mitchell et al. 2013). Relatively, little attention has been paid to identify the tropospheric precursors of SSWs, even when they have the potential benefit of improving the predictability of SSWs and hence, the lead time of the SSW-related anomalies in extended-range weather forecasts (e.g., Cohen and Jones 2011).

Several forcings affect the mean winter state of the $\mathrm{NH}$ polar vortex, such as El Niño-Southern Oscillation (ENSO), the quasi-biennial oscillation (QBO), volcanic 
eruptions, or the 11-yr solar cycle (e.g., Holton and Tan 1980; Robock and Mao 1992; García-Herrera et al. 2006; Matthes et al. 2006). In the last years a renewed interest has emerged to examine the role of ENSO. Observational and modeling studies reported an ENSO influence on the NH polar stratosphere, whereby the polar vortex is warmer and more perturbed during El Niño (EN) winters, with opposite but weaker responses occurring during La Niña (LN) (e.g., Sassi et al. 2004; Free and Seidel 2009; Calvo et al. 2010). The underlying mechanism is thought to involve an ENSO-forced extratropical response in the troposphere that modulates the upward planetary wave flux into the winter stratosphere and the polar vortex strength via planetary wave dissipation (e.g., García-Herrera et al. 2006; Manzini et al. 2006). Furthermore, modeling results suggested a tendency for SSWs to occur preferentially during EN winters (Taguchi and Hartmann 2006), as it would be expected from the seasonal mean effect of EN on the polar vortex. However, recently, Butler and Polvani (2011) found that, for the 1948-2010 period of the National Centers for Environmental Prediction-National Center for Atmospheric Research (NCEP-NCAR) reanalysis (Kalnay et al. 1996), SSWs occur with approximately equal frequency during EN and LN. Despite the limited sample size of the observational record, this result stresses the importance of both ENSO phases for the occurrence of SSWs.

On the other hand, the fact that SSWs can occur with relative independence from the seasonal mean state of the polar stratosphere highlights the relevance of subseasonal processes in driving SSWs (e.g., Quiroz 1986). Among them, tropospheric blocking (i.e., the breakdown of the prevailing tropospheric westerly flow by persistent anticyclones) has been identified as a potential precursor of SSWs (Martius et al. 2009; Castanheira and Barriopedro 2010; Woollings et al. 2010; Nishii et al. 2011). Using reanalysis data, Taguchi (2008) did not find statistically significant connections between blocks and SSWs; this was attributed to the fact that blocking is a more frequent phenomenon than SSWs. However, while not all blocks lead to SSWs, SSWs tend to be preceded by blocks. Thus, it is unclear whether there are specific signatures in blocking or in the SSWs themselves that promote the otherwise weak linkage between them. Recent studies have reported that the blocking precursors of SSWs occur over different regions depending on the type of SSW; hence, significant associations between blocking and SSWs only arise when SSWs are stratified into splitting and displacement cases (Martius et al. 2009; Castanheira and Barriopedro 2010). The growing body of evidence suggests that the blockinginduced perturbations in tropospheric planetary-scale waves of wavenumber 1 (hereafter wave 1) and wavenumber 2 (hereafter wave 2) depend on the blocking location, and in turn influence the type of SSW, with wave 1 being more related to displacement events and wave 2 related to splitting SSWs. There are also different precursors of splitting and displacement SSWs at monthlyto-seasonal time scales (Cohen and Jones 2011).

In this study, we examine the ENSO influence on the tropospheric blocking precursors of SSWs and on several features of the SSWs such as the type or the relative contribution of different wavenumbers. The paper is structured as follows. Section 2 describes the data and methods, which include the identification of ENSO events, the definition and diagnosis of SSWs, a description of the blocking algorithm, and the statistical analyses performed in this study. The main results are presented in section 3, which addresses the ENSO influence on the blocking precursors of SSWs and on the characteristics of the SSWs. To interpret the ENSO differences, we also explore the overall blocking response to ENSO and the mean blocking effect on the polar vortex depending on the ENSO phase. The main conclusions are summarized in section 4.

\section{Data and methods}

\section{a. SSWs characteristics}

The study was conducted for the 1958-2010 period by using data from the 40-yr European Centre for MediumRange Weather Forecasts (ECMWF) Re-Analysis (ERA-40; 1957/58-2001/02; Uppala et al. 2005) and the ECMWF Interim Re-Analysis (ERA-Interim; 2002/032009/10; Dee et al. 2011). Similar results were found for the NCEP-NCAR reanalysis and for the 1957/58-2001/02 period of the ERA-40. The list of SSWs and their classification into displacement and splitting events were taken from Charlton and Polvani (2007) for the 1957/58-2001/ 02 period of ERA-40 and from Cohen and Jones (2011) for the 2002/03-2009/10 period of the NCEP-NCAR reanalysis. Both used the same definition and criteria to identify SSWs (see Charlton and Polvani 2007): SSWs were detected as isolated episodes of zonal mean zonal wind reversal at $10 \mathrm{hPa}$ and $60^{\circ} \mathrm{N}$ during the extended NH winter season [November-March (NDJFM)]. There are 36 SSWs in the 1958-2010 period. The central dates of the SSWs (i.e., the first day of easterly wind) are shown in Table 1. It should be stressed that Cohen and Jones (2011) employed data from the NCEP-NCAR reanalysis but they reported no differences in the identification of cases or in the type of SSWs between the NCEP-NCAR and the ERA-Interim reanalyses. However, we noted some minor discrepancies in the central dates of the 
TABLE 1. Central dates (i.e., the first day with easterly winds, as diagnosed from 1200 UTC zonal mean zonal wind data at $10 \mathrm{hPa}$ and $60^{\circ} \mathrm{N}$ ), type [splitting (S) and displacement (D)], preconditioned wavenumber [wave $1\left(\mathrm{~W}_{1}\right)$ and wave $2\left(\mathrm{~W}_{2}\right)$ ], and the associated winter ENSO phase of SSWs in the 1958-2010 period. The SSWs of the ERA-40 period 1958-2002 are taken from Charlton and Polvani (2007). For the 2003-10 period, the SSWs are from Cohen and Jones (2011), but reassigning their central dates to those detected with the ERA-Interim.

\begin{tabular}{|c|c|c|c|c|}
\hline Reanalysis & Central date* & Type & Wave & ENSO phase ** \\
\hline \multirow[t]{29}{*}{ ERA-40 } & 31 Jan 1958 & $\mathrm{~S}$ & $\mathrm{~W}_{1}$ & EN \\
\hline & 15 Jan 1960 & $\mathrm{D}$ & $\mathrm{W}_{1}$ & $\mathrm{NE}$ \\
\hline & 28 Jan 1963 & S & $\mathrm{W}_{2}$ & LN \\
\hline & 16 Dec 1965 & $\mathrm{D}$ & $\mathrm{W}_{1}$ & EN \\
\hline & 23 Feb 1966 & S & $\mathrm{W}_{1}$ & EN \\
\hline & 7 Jan 1968 & $\mathrm{~S}$ & $\mathrm{~W}_{2}$ & $\mathrm{LN}$ \\
\hline & 28 Nov 1968 & $\mathrm{D}$ & $\mathrm{W}_{1}$ & EN \\
\hline & 13 Mar 1969 & $\mathrm{D}$ & $\mathrm{W}_{1}$ & EN \\
\hline & 1 Jan 1970 & $\mathrm{D}$ & $\mathrm{W}_{1}$ & $\mathrm{NE}$ \\
\hline & 18 Jan 1971 & S & $\mathrm{W}_{2}$ & LN \\
\hline & 19 Mar 1971 & $\mathrm{D}$ & $\mathrm{W}_{1}$ & $\mathrm{LN}$ \\
\hline & 31 Jan 1973 & S & $\mathrm{W}_{1}$ & $\mathrm{EN}$ \\
\hline & 9 Jan 1977 & $\mathrm{~S}$ & $\mathrm{~W}_{1}$ & EN \\
\hline & 22 Feb 1979 & $\mathrm{~S}$ & $\mathrm{~W}_{2}$ & $\mathrm{NE}$ \\
\hline & 29 Feb 1980 & $\mathrm{D}$ & $\mathrm{W}_{1}$ & $\mathrm{NE}$ \\
\hline & 4 Mar 1981 & $\mathrm{D}$ & $\mathrm{W}_{1}$ & $\mathrm{NE}$ \\
\hline & 4 Dec 1981 & $\mathrm{D}$ & $\mathrm{W}_{1}$ & $\mathrm{NE}$ \\
\hline & 24 Feb 1984 & $\mathrm{D}$ & $\mathrm{W}_{1}$ & $\mathrm{NE}$ \\
\hline & 1 Jan 1985 & $\mathrm{~S}$ & $\mathrm{~W}_{2}$ & $\mathrm{LN}$ \\
\hline & 23 Jan 1987 & $\mathrm{D}$ & $\mathrm{W}_{1}$ & $\mathrm{EN}$ \\
\hline & 7 Dec 1987 & S & $\mathrm{W}_{1}$ & EN \\
\hline & 14 Mar 1988 & S & $\mathrm{W}_{1}$ & $\mathrm{EN}$ \\
\hline & 21 Feb 1989 & $\mathrm{~S}$ & $\mathrm{~W}_{2}$ & $\mathrm{LN}$ \\
\hline & 15 Dec 1998 & $\mathrm{D}$ & $\mathrm{W}_{1}$ & $\mathrm{LN}$ \\
\hline & 26 Feb 1999 & S & $\mathrm{W}_{1}$ & LN \\
\hline & 20 Mar 2000 & $\mathrm{D}$ & $\mathrm{W}_{2}$ & $\mathrm{LN}$ \\
\hline & 11 Feb 2001 & $\mathrm{~S}$ & $\mathrm{~W}_{1}$ & $\mathrm{LN}$ \\
\hline & 30 Dec 2001 & $\mathrm{D}$ & $\mathrm{W}_{1}$ & $\mathrm{NE}$ \\
\hline & 17 Feb 2002 & $\mathrm{D}$ & $\mathrm{W}_{1}$ & $\mathrm{NE}$ \\
\hline \multirow[t]{7}{*}{ ERA-Interim } & 18 Jan 2003 & $\mathrm{~S}$ & $\mathrm{~W}_{1}$ & EN \\
\hline & 5 Jan 2004 & $\mathrm{D}$ & $\mathrm{W}_{1}$ & $\mathrm{NE}$ \\
\hline & 21 Jan 2006 & $\mathrm{~S}$ & $\mathrm{~W}_{1}$ & $\mathrm{LN}$ \\
\hline & 24 Feb 2007 & $\mathrm{D}$ & $\mathrm{W}_{1}$ & $\mathrm{EN}$ \\
\hline & 22 Feb 2008 & $\mathrm{D}$ & $\mathrm{W}_{2}$ & $\mathrm{LN}$ \\
\hline & 24 Jan 2009 & $\mathrm{~S}$ & $\mathrm{~W}_{2}$ & $\mathrm{LN}$ \\
\hline & 9 Feb 2010 & $S$ & $\mathrm{~W}_{1}$ & EN \\
\hline
\end{tabular}

* An additional event was detected on 23 Mar 2010 with the ERAInterim and the NCEP-NCAR reanalyses. This episode was not considered by Cohen and Jones (2011). We disregarded it to avoid a subjective judgment of the type of SSW and possibly using different classification criteria across the SSWs.

** The classification of ENSO events is only faintly different from that provided by Butler and Polvani (2011). The discrepancies are due to small differences in the criteria for the classification of ENSO events and the truncation of the N3.4 index.

events and thus, we chose the central dates detected with the ERA-Interim.

According to Bancalá et al. (2012), the classification of SSWs into displacement and splitting events is based on a suite of criteria that tends to be biased toward the postwarming phase of the SSW. As we are interested here in precursors of SSWs, we also categorized SSWs into wave- 1 and wave- 2 events, depending on the planetary wave activity for a period before the SSW, herein taken as the $[-10,0]$-day interval (the day 0 refers to the central date of the SSW). This classification was done by applying a zonal Fourier decomposition of the 50-hPa geopotential height field at $60^{\circ} \mathrm{N}$ on a daily basis, and retaining the amplitudes of wavenumbers $1\left(Z_{1}\right)$ and $2\left(Z_{2}\right)$ for the $[-10,0]$-day period before each SSW.

SSWs were classified into wave- 1 and wave- 2 events according to the following criteria: (i) the SSW was defined as a wave- 2 event if $\left[Z_{2}\right] \geq\left[Z_{1}\right]$ (the brackets denote the averaged amplitude for the $[-10,0]$-day period before the SSW) or if $Z_{2}-Z_{1} \geq 200 \mathrm{gpm}$ at least for one day within the $[-10,0]$-day period before the SSW; and (ii) the SSW was defined as a wave-1 event if $\left[Z_{1}\right]>\left[Z_{2}\right]$ and $Z_{2}-Z_{1}<200 \mathrm{gpm}$ for all days of the [-10,0]-day period before the SSW. This method is similar to (but simpler than) that proposed by Bancalá et al. (2012). The second condition in (i) and (ii) (i.e., that referring to $Z_{2}-Z_{1}$ ) was imposed to avoid filtering wave-2 SSWs by the smoothing inherent to the temporal average. However, this criterion was found to be of secondary importance (it only affected the classification of two SSWs), since most SSWs are unequivocally determined by the value of $\left[Z_{2}\right]-\left[Z_{1}\right]$. The choice of the 50 -hPa pressure level is justified because it is near the climatological maximum of $Z_{2}$ in a vertical profile, and the threshold of $200 \mathrm{gpm}$ approximately coincides with the 90th percentile of the $Z_{2}-Z_{1}$ time series. The resulting catalog of wave- 1 and wave-2 SSWs is shown in Table 1 , together with the classification of SSWs in splitting or displacement types, and is similar to that provided by Bancalá et al. (2012, their Table 1) for the 1958-2002 period. In fact, the conclusions of this study remain if we use their classification for wave- 1 and wave-2 SSWs.

In addition to $Z_{1}$ and $Z_{2}$, we also computed daily values of the meridional eddy heat flux at $100 \mathrm{hPa}\left(\overline{v^{\prime} T^{\prime}}\right)$ averaged over $45^{\circ}-75^{\circ} \mathrm{N}$ (and weighted by the cosine of the latitude), which provides a measure of the planetary wave activity flux entering the stratosphere (e.g., Polvani and Waugh 2004). This was also done for wave 1 and wave 2 separately (i.e., $\overline{v_{1}^{\prime} T_{1}^{\prime}}$ and $\overline{v_{2}^{\prime} T_{2}^{\prime}}$ ) after applying a fast Fourier transform to the daily fields of $v^{\prime}$ and $T^{\prime}$ at $100 \mathrm{hPa}$ (e.g., Newman and Nash 2000). In the above expressions $v^{\prime}$ and $T^{\prime}$ are the deviations from the zonal mean, $v_{i}^{\prime}$ and $T_{i}^{\prime}$ are the amplitudes of the $i$ th wave component of the meridional wind and temperature, and the overbar denotes the zonal mean. Finally, we computed the daily anomalies of the poleward eddy heat fluxes. Throughout this paper, daily anomalies are 
always defined with reference to the seasonal cycle by subtracting the daily climatology. Fluctuations of the poleward eddy heat flux can be interpreted in terms of variability in the upward planetary wave propagation (e.g., Polvani and Waugh 2004), with positive (negative) anomalies of $\overline{v_{1}^{\prime} T_{1}^{\prime}}$ and $\overline{v_{2}^{\prime} T_{2}^{\prime}}$ representing an enhanced warming (cooling) of the lowermost stratosphere because of increased (decreased) upward propagation of wave 1 and wave 2 , respectively.

\section{b. ENSO events}

ENSO winters were defined for the 1958-2010 period according to the NDJFM average of the Niño-3.4 index (hereafter N3.4). The Niño-3.4 index is the regional $\left(5^{\circ} \mathrm{S}-5^{\circ} \mathrm{N}, 170^{\circ} \mathrm{E}-120^{\circ} \mathrm{W}\right)$ monthly-mean sea surface temperature anomaly with reference to 1971-2000 (the monthly series was extracted online at http://www.cpc. ncep.noaa.gov/). Similar to Butler and Polvani (2011), $\mathrm{EN}, \mathrm{LN}$ and neutral (NE) winters were identified when $\mathrm{N} 3.4 \geq 0.50^{\circ} \mathrm{C}, \mathrm{N} 3.4 \leq-0.50^{\circ} \mathrm{C}$, and $-0.5<\mathrm{N} 3.4<$ $0.5^{\circ} \mathrm{C}$, respectively. This leads to a balanced distribution of ENSO phases, with $17 \mathrm{EN}, 17 \mathrm{LN}$, and $19 \mathrm{NE}$ winters in the 53-winter record. We note that different thresholds for N3.4 [those used in Butler and Polvani (2011)] yielded similar results to those reported in this paper. Table 1 lists the ENSO phase corresponding to each SSW. Out of the 36 SSWs of the 1958-2010 period, 13 SSWs occurred during EN winters (EN SSWs), 13 events occurred during LN winters (LN SSWs), and $10 \mathrm{SSWs}$ occurred during NE winters.

\section{c. Blocking detection}

Daily blocks were detected as large-scale areas $(\geq 1.8 \times$ $10^{6} \mathrm{~km}^{2}$ ) with anomalies of the $500-150-\mathrm{hPa}$ vertically integrated potential vorticity (PV) below $-1.3 \mathrm{PVU}$ (1 PVU $=10^{-6} \mathrm{~K} \mathrm{~kg}^{-1} \mathrm{~m}^{2} \mathrm{~s}^{-1}$; the 10th percentile of the PV distribution). A tracking algorithm was employed to follow the daily evolution of the $2 \mathrm{D}$ blocking areas and to ensure quasi stationarity and persistence of the blocking events [see Castanheira and Barriopedro (2010) for further details]. The blocking detection scheme is identical to that employed by Martius et al. (2009), except that we adapted it to daily data, instead of the 6-hourly resolution used therein. To test the sensitivity of the results with respect to the blocking definition, we repeated the analyses with a novel blocking algorithm based on geopotential height data at $500 \mathrm{hPa}$ (Barriopedro et al. 2010), without finding remarkable differences.

\section{d. Composite methods}

To address the ENSO influence on blocking we performed 2D composites of blocking frequency and persistence for EN and LN winters. The blocking frequency was assessed by evaluating for each grid point the number of days that a blocking pattern was detected. Blocking persistence was computed by identifying all blocking episodes that affected (during at least 1 day) a given grid point and then averaging their total duration and assigning this value to that grid point. The statistical significance (against climatology) of the EN and LN composites was assessed with a Monte Carlo test of 1000 random subsamples of winters with the same size as the number of EN and LN winters and random years of occurrence.

The blocking precursors of SSWs are identified by performing $2 \mathrm{D}$ composites of the blocking frequency for the $[-10,0]$-day period before the central day of each SSW. This time scale is close to the typical duration of blocking and to the lead time of numerical weather forecasts for which SSWs are predictable (Gerber et al. 2009; Marshall and Scaife 2010), although the results are not highly sensitive to the choice of the temporal window (e.g., a [-20, 0]-day interval yields similar results). The same analysis was performed for SSWs stratified according to the ENSO phase. In all these cases, significant differences with respect to climatology were assessed with a Monte Carlo test of 1000 members, each one containing the same number of cases and dates as the SSWs of each composite but with random years of occurrence (Taguchi 2008; Martius et al. 2009; Castanheira and Barriopedro 2010).

Finally, to interpret the ENSO differences in the blocking precursors, we assessed the wave activity at the polar stratosphere induced by blocks, by computing 2D composites of $Z_{1}, Z_{2}$, and poleward eddy heat fluxes following the occurrence of blocking at a given grid point. To do so, local blocking occurrences were identified from those winter days when a $2 \mathrm{D}$ blocking pattern affected a given grid point. For each one of these local blocking episodes, we computed the average values of $Z_{1}, Z_{2}$, and heat fluxes anomalies for the 10-day period following the initiation (day 0) of the local blocking event at the given grid point (as in Nishii et al. 2011). The length of this time interval approximately corresponds to the time for tropospheric anomalies to propagate to the stratosphere (e.g., Limpasuvan et al. 2004). These dynamical fields were then composited at each grid point of the NH for all local blocking episodes of the extended winter. The significance was assessed with a Monte Carlo test of 1000 trials in which the same number of cases of each grid point is chosen with random years of occurrence, but keeping the days and months of the blocking events intact. Similar composite analyses were also derived for EN and LN winters, separately. 


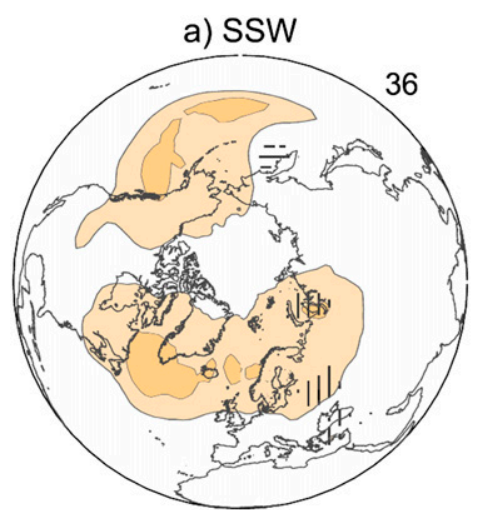

d) EN-LN SSW

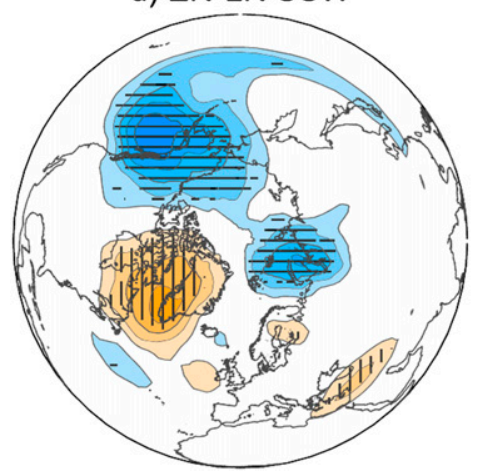

b) EN SSW

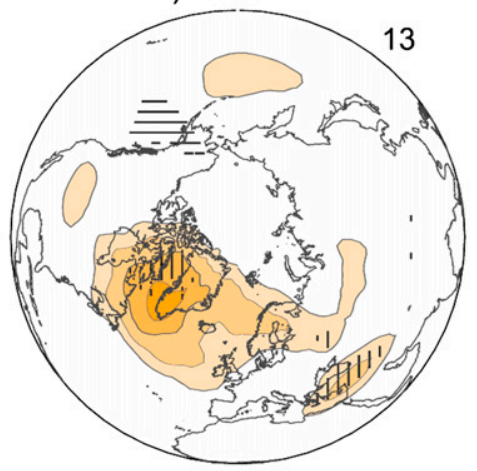

e) SSW displ.-split

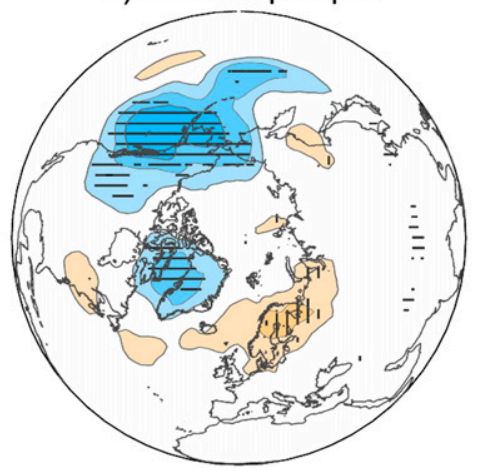

c) LN SSW

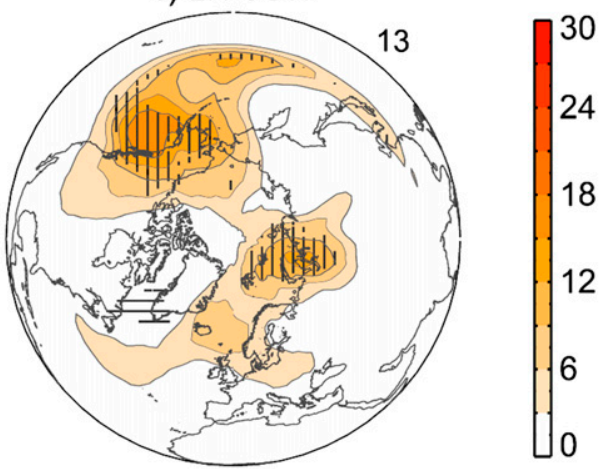

f) SSW w1-w2

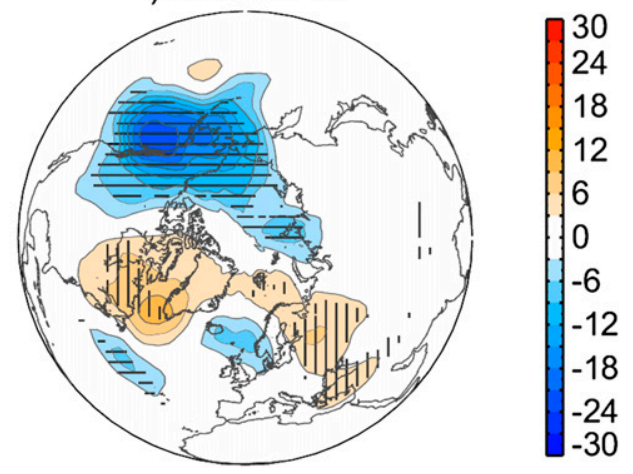

FIG. 1. Composites of blocking precursors of SSWs for (a) all, (b) EN, (c) LN winters, and the composite difference of the blocking precursors for (d) EN minus LN SSWs, (e) displacement minus splitting SSWs, and (f) wave-1 minus wave-2 SSWs. Blocking precursors are identified from the blocking frequency for the $[-10,0]$-day period before the central date of SSWs. The blocking frequency is expressed as the percentage of time (over the 11-day period) during which a blocking was detected at each grid point. The number of SSWs entering into the composites is shown in the top-right corner of each panel. Vertical (horizontal) hatched areas indicate regions with blocking activity significantly above (below) climatology at the $95 \%$ confidence level as derived from a 1000-trial Monte Carlo test. Note that the detection of a significantly low blocking frequency $(p<5 \%)$ in (a)-(c) is hampered in regions where the climatological blocking activity is low.

\section{Results}

\section{a. ENSO modulation of blocking precursors of SSWs}

First, the characteristic blocking precursors of SSWs and their spatial differences with the ENSO phase are explored. Figures $1 \mathrm{a}-\mathrm{c}$ show the composites of blocking $[-10,0]$-day prior to SSWs as derived from all winters and from EN and LN winters separately. The results indicate weak links between blocking and SSWs when SSWs are considered altogether (Fig. 1a). However, a more robust signal arises if SSWs are stratified by the ENSO phase, with large differences in the location of the blocking precursors between EN and LN. During EN (Fig. 1b), SSWs tend to be preceded by blocking occurrence over the Euro-Atlantic sector (although the signal is only significant over western Atlantic), while eastern Pacific and, secondarily, northern Siberia blocking are significantly the preferred precursors of SSWs during LN (Fig. 1c). Comparatively, the spatial pattern during NE winters shows some blocking activity over the Euro-Atlantic sector, although the signal is only statistically significant over small regions of eastern Europe (not shown). Interestingly, the behavior of blocking activity over the Pacific and the Atlantic is reversed during opposite ENSO phases: Pacific blocking frequency is significantly increased (cf. climatology) before LN SSWs and reduced prior to EN SSWs, and the opposite occurs for Atlantic blocking. This is reflected in the composite difference between EN and LN (Fig. 1d). These results suggest an ENSO influence on blocking precursors of SSWs, with a clear spatial preference for specific blocking precursors depending on the ENSO phase. Therefore, in the following, we will mostly focus on EN and LN phases, disregarding SSWs occurring during NE winters.

On the other hand, previous studies have reported a spatial dependence of the blocking precursors on the type of SSWs (see section 1). According to Martius et al. (2009), splitting events are preceded by either Pacific 
a) SSW-displ.

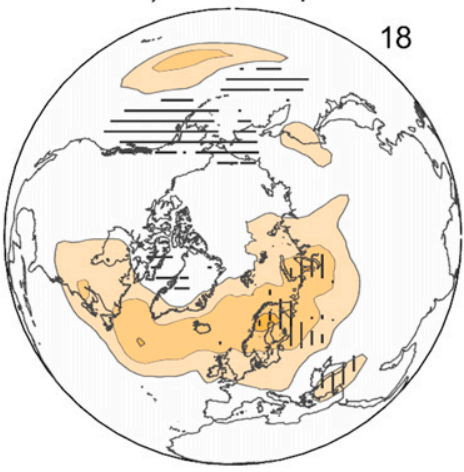

b) SSW-split

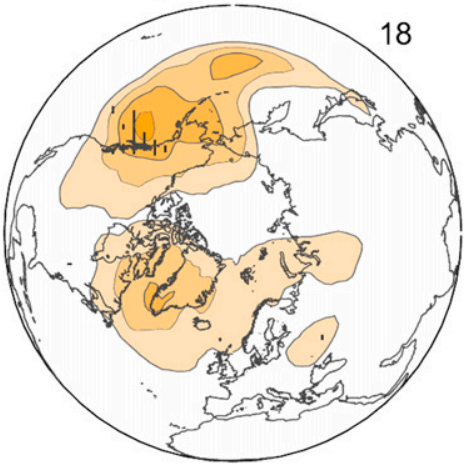

c) EN SSW-displ.

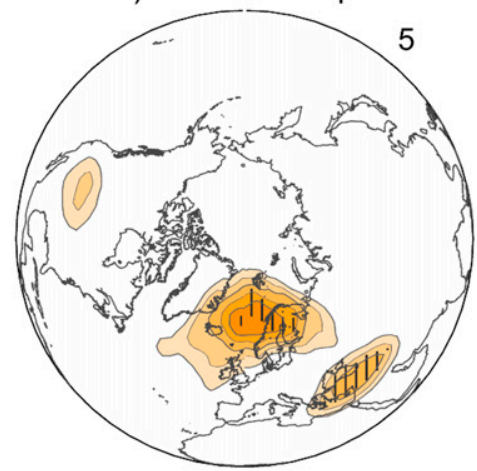

d) EN SSW-split

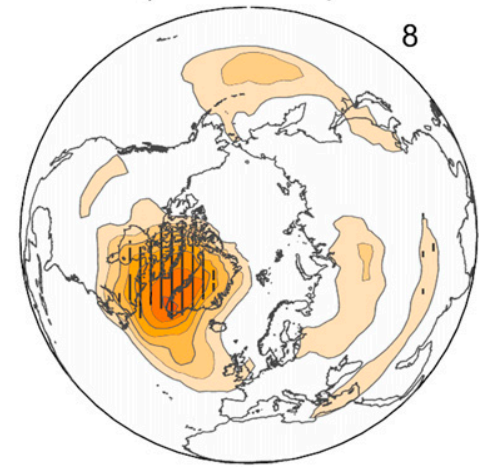

e) LN SSW-displ.

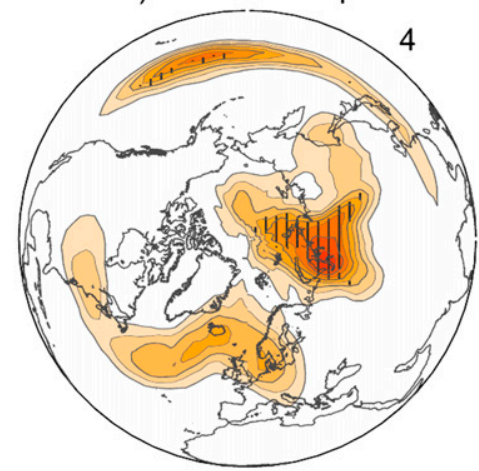

f) LN SSW-split

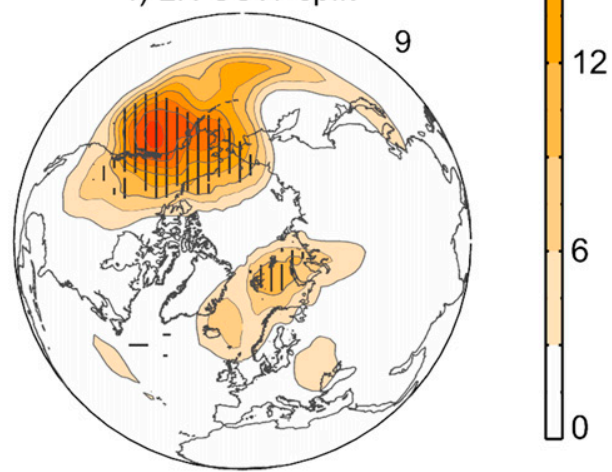

FIG. 2. Composites of blocking precursors of (top) displacement and (bottom) splitting SSWs for (a),(b) all winters; (c),(d) EN winters; and (e),(f) LN winters. See the caption of Fig. 1 for details.

blocks alone or Atlantic and Pacific blocks simultaneously, while displacement SSWs are associated with a lack of Pacific blocking and enhanced blocking frequency over the Euro-Atlantic/Euro-Asian sector. This is reproduced in Figs. $2 \mathrm{a}$ and $2 \mathrm{~b}$, which show the composited rate of blocking frequency for the $[-10,0]$-day period preceding displacement and splitting SSWs, respectively. SSWs that occur during EN and LN share some of their blocking precursors with those of displacement and splitting SSWs, respectively (cf. Figs. $1 \mathrm{~b}$ and $1 \mathrm{c}$ ). To explore if this is due to an EN (LN) preference for displacement (splitting) SSWs, we have computed the frequency of SSWs and their types for each ENSO phase (Fig. 3a). Using the NCEP-NCAR reanalysis, Butler and Polvani (2011) obtained that SSWs occur with similar frequencies during EN and LN and are less common during NE. Our results (dark bars in Fig. 3a, top plot) confirm this finding for a different reanalysis. A breakdown of the results by the type of SSW sheds more light on the ENSO-SSW relationship. For the SSW definition employed herein, splitting events tend to occur more often during LN than in the climatology, whereas EN winters are not biased toward any particular type of SSW. It is also remarkable that the reduction of SSWs during
NE reported by Butler and Polvani (2011) arises from an almost complete lack of splitting SSWs ( $p>95 \%$ ), just one event in 19 winters. Similar results are obtained from the NCEP-NCAR reanalysis and for small changes in the classification of ENSO years, although the small sample size of typed SSWs compromises the statistical robustness of our results, which should therefore be taken with caution.

The results of Fig. 3 indicate that, although there is some tendency for splitting SSWs during LN, EN does not show any preference for a type of SSW. In fact, the difference in SSW frequency between EN and LN winters is not statistically significant for any type of SSWs (Fig. 3a, bottom plot). Hence, the differences in the blocking patterns between EN and LN shown in Figs. 1b,c cannot be attributed to a distinctive type of SSW during $\mathrm{EN}$ and LN. A comparison of the relative importance of the effects of ENSO and the type of SSWs on the blocking precursors of SSWs is shown in Figs. 1d,e. The differences between EN and LN (Fig. 1d) are larger than those between splitting and displacement SSWs (Fig. 1e), indicating that the ENSO effect on the spatial blocking precursors dominates over the effect due to the type of SSW. 
a) SSW type
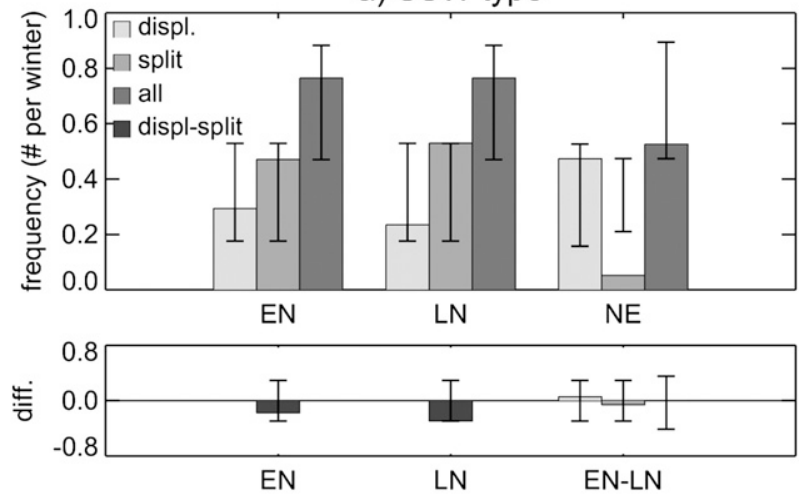

b) SSW wave
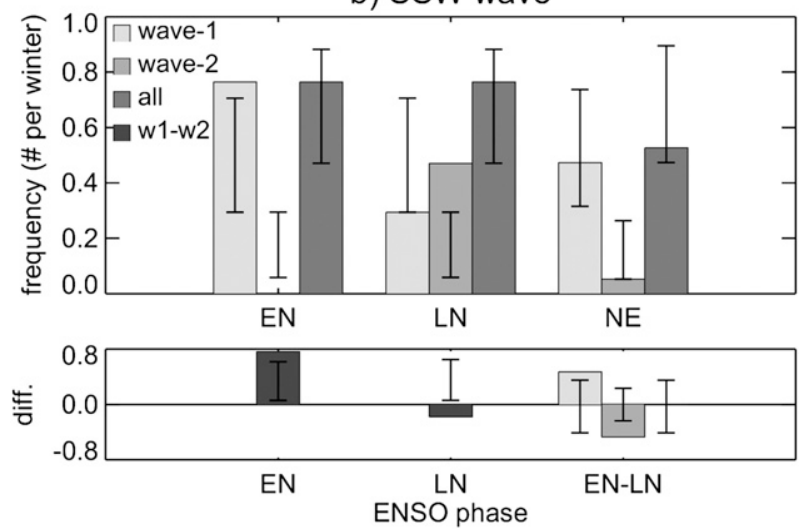

FIG. 3. (a) Relative frequency of SSWs and their types (splitting and displacement) according to the ENSO phase, shown in the top plot. The frequency difference between displacement and splitting SSWs during EN and LN (dark bars), and the EN minus LN difference of all SSWs and their types are shown in the bottom plot. (b) As in (a), but for wave-1 and wave-2 SSWs. The frequencies are computed as a conditional probability (i.e., number of SSWs during a given ENSO phase divided by the number of winters with that ENSO phase). Whiskers indicate the 5th-95th intervals derived from a Monte Carlo test of 5000 random subsamples with the same size as the number of EN, LN, and NE winters.

Up to now, we have reported an ENSO modulation of the blocking precursors of SSWs. Next, we explore the ENSO influence on the blocking precursors of displacement and splitting SSWs separately. To do so, composites of blocking precursors were computed for displacement and splitting SSWs and for each ENSO phase, and the results are shown in Figs. 2c-f. Note that the sample size is small in some of these composites, in particular those of displacement SSWs. Splitting SSWs that occur during EN (Fig. 2d) and LN (Fig. 2f) display major differences in their blocking precursors. While Atlantic blocking tends to precede splitting events during EN, Pacific blocks occur predominantly before $\mathrm{LN}$ vortex splits. As a consequence, the co-occurrence of blocking over the Pacific and the Atlantic before splitting SSWs (Fig. 2b) is no longer significant when vortex splits are stratified by the ENSO phase. Instead, splits of EN and LN winters are significantly associated with blocking precursors over different basins. Note also that NE cases would not distort this conclusion, as there is only one splitting SSW during NE winters. Different from splitting SSWs, the composites for displacement SSWs do not exhibit opposite blocking patterns between EN and LN (Figs. 2c,e). Still, there are some remarkable differences, the most striking one corresponding to the tendency for displacement SSWs to be preceded by Siberian blocks during LN (Fig. 2e) and by European blocks during EN (Fig. 2c). The above results reveal an ENSO signal on the spatial blocking precursors of splitting and displacement SSWs. Thus, the relationship between blocking and the type of SSW reported in previous studies is not univocal, but is modulated by ENSO.

\section{b. Stratospheric response to blocking and its ENSO modulation}

To understand the underlying dynamics behind the ENSO modulation of the blocking precursors of SSWs, we explore the mean blocking effect on the polar stratosphere and its dependence with the ENSO phase, regardless of whether or not blocking leads to SSWs. To assess the wave influx that reaches the lower stratosphere induced by blocking episodes, we performed composites of anomalous zonal mean poleward eddy heat flux at $100 \mathrm{hPa}$ averaged over $45^{\circ}-75^{\circ} \mathrm{N}\left(\overline{v^{\prime} T^{\prime}}\right)$ and of its wave components $\overline{v_{1}^{\prime} T_{1}^{\prime}}$ and $\overline{v_{2}^{\prime} T_{2}^{\prime}}$ separately, for the [0, 10]-day mean period after the occurrence of a block at each grid point of the NH (Fig. 4).

Overall, there are significant positive values of the total eddy heat flux over most of the Euro-Atlantic sector and negative values over western Pacific (Fig. 4a). As a consequence, Euro-Atlantic (western Pacific) blocks tend to precede a warmer (colder) polar stratosphere because of enhanced (suppressed) upward wave propagation and subsequent dissipation. This agrees with previous studies reporting links between the strength of the polar vortex and precursor tropospheric anomalies over the European or the North Pacific sector (e.g., Garfinkel et al. 2010). Thus, the blocking influence on the polar stratosphere depends on the geographical location of the block. This regional-dependent influence of blocking on the polar vortex can be explained by the relative location of the block with respect to the geographical phase of the stationary planetary waves of wavenumber 1 and 2 (e.g., Castanheira and Barriopedro 2010; Nishii et al. 2011).

By separating the total poleward heat flux into wave-1 (Fig. 4b) and wave-2 (Fig. 4c) components, it is found that the net response of the polar vortex to blocking mainly 
a) heat flux

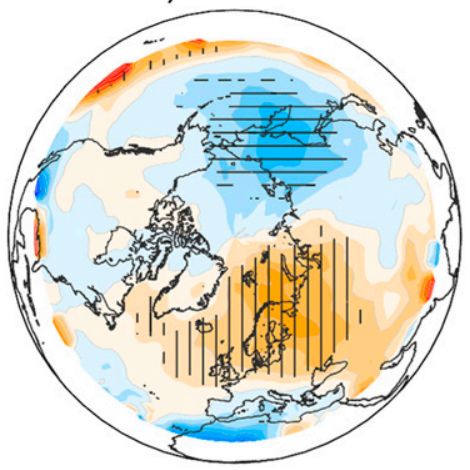

d) EN heat flux

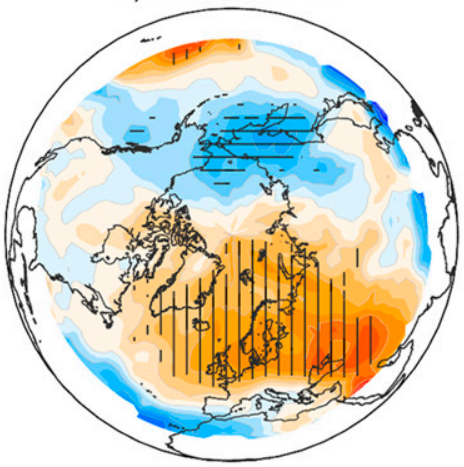

g) LN heat flux

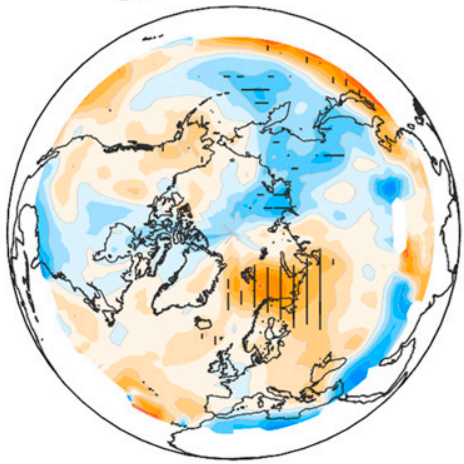

b) heat flux-w1

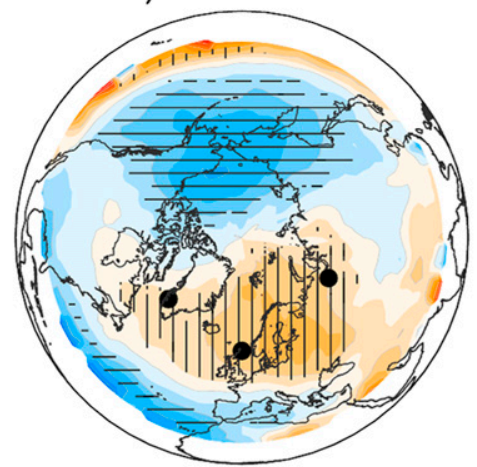

e) EN heat flux-w1

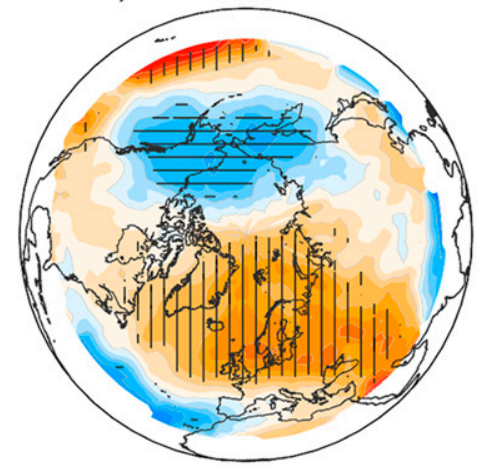

h) LN heat flux-w1

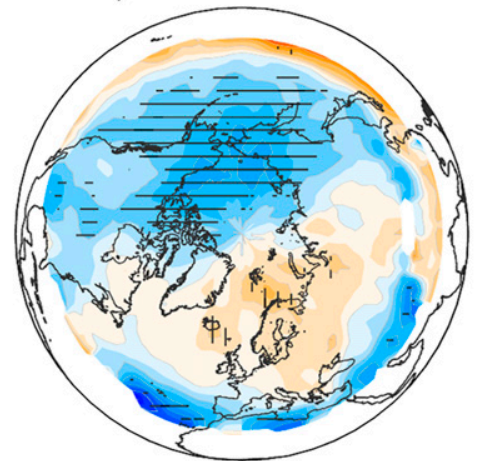

c) heat flux-w2

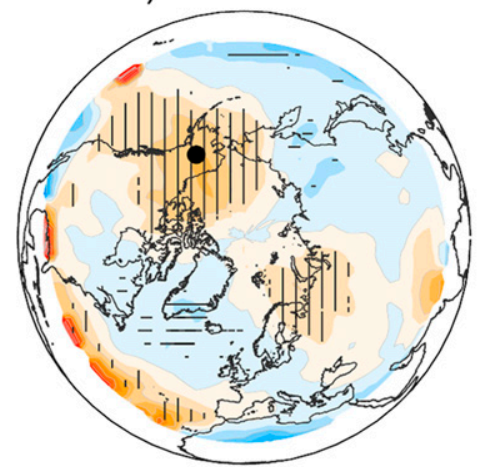

f) EN heat flux-w2

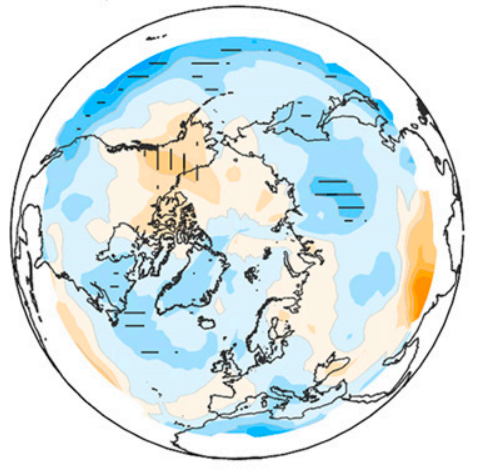

i) LN heat flux-w2

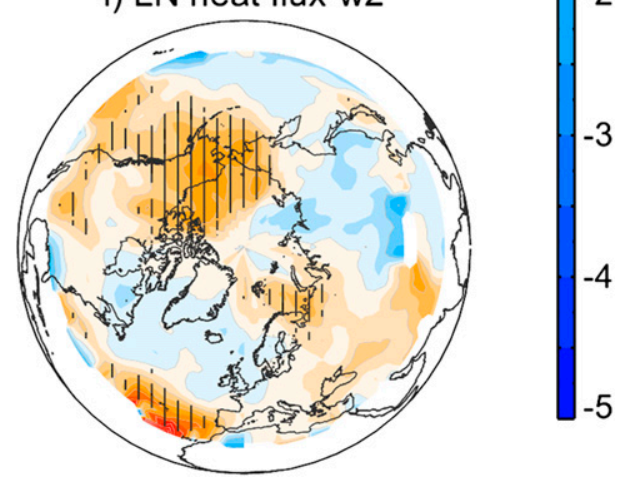

FIG. 4. Composites of zonal mean meridional eddy heat flux anomalies $\left(\mathrm{K} \mathrm{m} \mathrm{s}^{-1}\right)$ at $100 \mathrm{hPa}\left(\overline{v^{\prime} T^{\prime}}\right)$ and of its wave-1 $\left(\overline{v_{1}^{\prime} T_{1}^{\prime}}\right)$ and wave-2 $\left(\overline{v_{2}^{\prime} T_{2}^{\prime}}\right)$ components averaged for $45^{\circ}-75^{\circ} \mathrm{N}$ and for the $[0,10]$-day period following the occurrence of blocking at each grid point of the $\mathrm{NH}$. The composites for (a)-(c) all, (d)-(f) EN, and (g)-(i) LN winters. The composites for (left) total, (center) wave-1, and (right) wave-2 heat fluxes. Positive (negative) values indicate a wave-driven warming (cooling) response of the lower stratosphere to local blocking occurrence. Vertical (horizontal) hatched areas identify those regions with poleward heat flux anomalies that are statistically significant above (below) normal at the $95 \%$ confidence level using a 1000-trial Monte Carlo test. Black dots in (b),(c) highlight selected locations.

arises from the contribution of wave 1 . However, and more importantly, there are different regional blocking influences on wave 1 and wave 2 (cf. Figs. $4 \mathrm{~b}$ and $4 \mathrm{c}$ ). This is further illustrated in Fig. 5, which shows zonal crosssection composites of anomalous wave- 1 and wave- 2 components of the geopotential height field induced by blocking at selected geographical locations. Overall, the wave amplitude of a given wavenumber is enhanced by blocking when the region of blocking coincides with the stationary ridges of that wavenumber in the mid- to upper troposphere, while the opposite occurs if the blocking pattern is collocated with the corresponding stationary troughs. The resulting amplified (weakened) patterns of the stationary waves are tilted westward with height, indicating an enhanced (suppressed) upward propagation of planetary-scale Rossby waves. A more detailed 


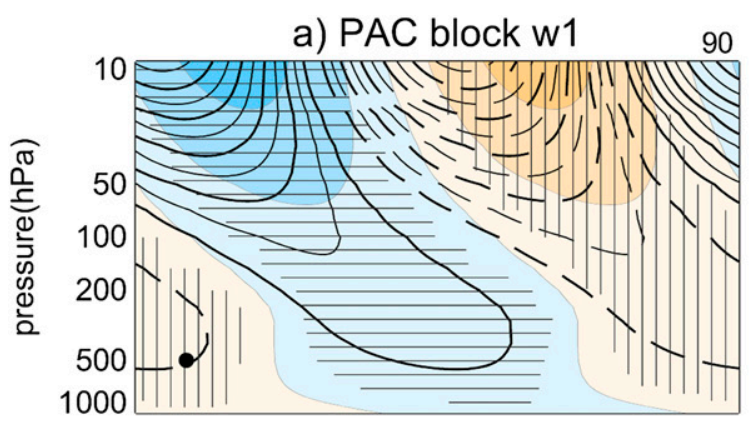

c) EUR block w1

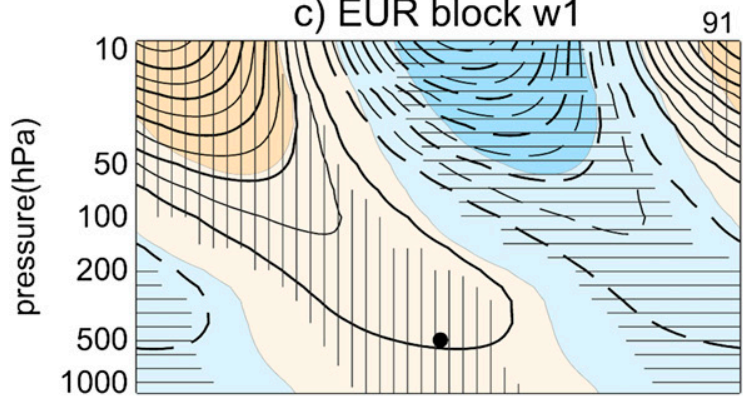

e) ATL block w1

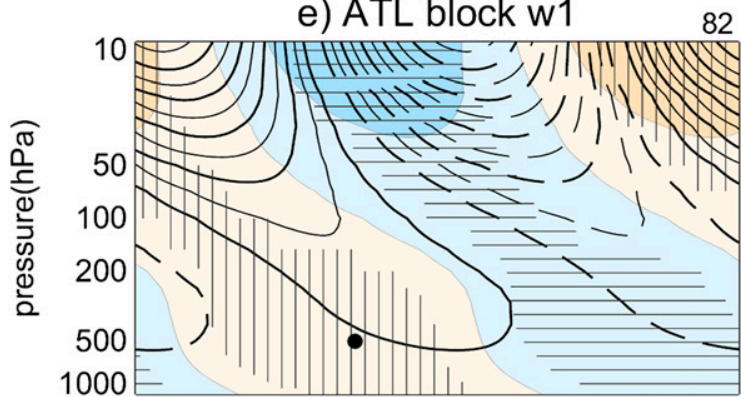

g) SIB block w1

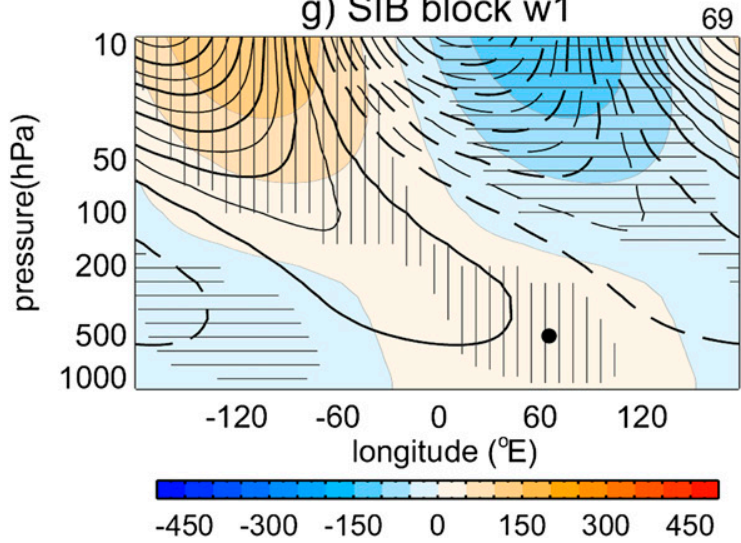

b) PAC block w2

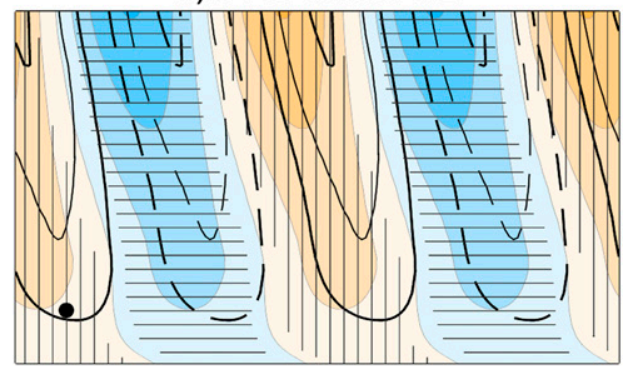

d) EUR block w2

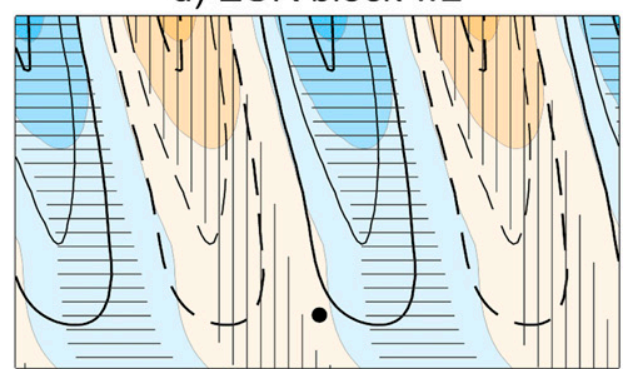

f) ATL block w2

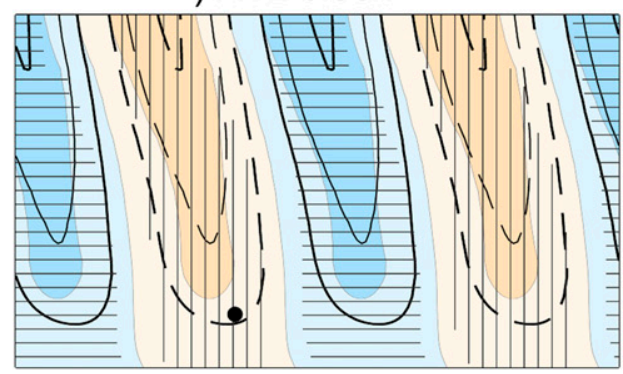

h) SIB block w2

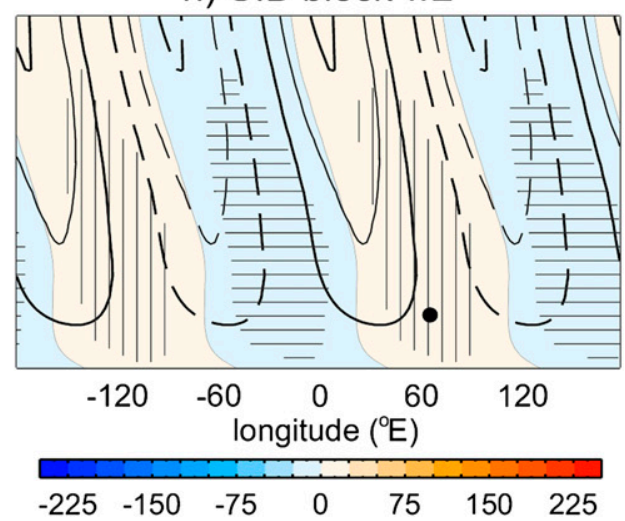

FIG. 5. Longitude-pressure cross-section composites of (left) wave-1 and (right) wave-2 anomalies (gpm, color shading) for the $[0,10]$-day period after the onset of blocking at selected locations (the blocking position is displayed with a black dot in each panel corresponding to locations in Figs. $4 \mathrm{~b}, \mathrm{c})$ for (a),(b) Pacific $\left(65^{\circ} \mathrm{N}, 150^{\circ} \mathrm{W}\right)$; (c),(d) European $\left(60^{\circ} \mathrm{N}, 0^{\circ}\right)$; (e),(f) Atlantic $\left(65^{\circ} \mathrm{N}, 50^{\circ} \mathrm{W}\right)$; and $(\mathrm{g}),(\mathrm{h})$ Siberian $\left(65^{\circ} \mathrm{N}, 65^{\circ} \mathrm{E}\right)$ blocks. Contour lines show the extended winter climatology with contour interval of $50 \mathrm{gpm}$ and solid (dashed) lines indicating positive (negative) values (the zero contour is omitted). The number of cases in each composite is shown at the top-right corner of the left-hand panels. Vertical (horizontal) hatched areas denote anomalous wave amplitudes that are significantly above (below) climatology at the $95 \%$ confidence level, after a 1000-trial Monte Carlo test. 
assessment performed at different time lags following blocking occurrence indicates that these wave perturbations appear almost immediately after the blocking episode and persist for several days, which suggests a relatively fast bottom-up influence (not shown).

Looking in detail at the regions of blocking precursor of SSWs, it is found that eastern Pacific blocks have opposite effects on wave 1 and wave 2 (Figs. 5a,b), resulting from a destructive (constructive) interference between the blocking anomaly and the stationary wave 1 (wave 2). The competing effects of eastern Pacific blocks on wave 1 and wave 2 are also observed in Figs. 4b,c, and imply a partial cancellation on the total eddy heat flux (cf. Figs. 4a and 4b,c) that could explain why some eastern Pacific blocks lead to significant perturbations of the polar vortex while others do not (Nishii et al. 2011). On the other hand, European (Figs. 5c,d) and Atlantic (Figs. 5e,f) blocks tend to enhance wave-1 propagation and suppress wave- 2 propagation, since these blocking regions are embedded in the Euro-Atlantic ridge of the stationary tropospheric wave 1 and relatively close to the stationary trough of wave 2 (see also Figs. 4b,c). Finally, the results for Siberian blocks (Figs. 5g,h) indicate an enhanced poleward heat flux in the lower stratosphere, mostly related to wave 1 , but also to wave 2 . Previous studies have already found that Siberian blocks can intensify the upward propagation of wave 1 and eventually cause a significant warming of the polar stratosphere (Nishii et al. 2011).

We next explore if the blocking effects over the polar vortex are sensitive to the ENSO phase. The composite of total eddy heat flux for blocks occurring during EN (Fig. 4d) is similar to that found for all winters, but with a stronger signal over the Euro-Atlantic sector, which includes the main precursor of SSWs during EN (Fig. 1b). In contrast, during LN (Fig. 4g), eastern Pacific and Siberia (the precursor regions of LN SSWs, Fig. 1c) are the main blocking regions that foster a warming the polar stratosphere, whereas the total poleward eddy heat flux following Euro-Atlantic blocks is considerably reduced. Therefore, the blocking regions that are more prone to weaken the polar vortex during EN and LN are also the corresponding locations of the blocking precursors of SSWs (Fig. 1). On the other hand, the comparison of the wave- 1 and wave- 2 heat flux components (Figs. 4b,c) and their respective composites during EN (Figs. 4e,f) and LN (Figs. 4h,i) indicates that the enhanced heat flux associated with Atlantic blocks during $\mathrm{EN}$ is due to wave 1 . In fact, the wave-1 activity following Atlantic blocks is strong enough to perturb significantly the polar stratosphere during EN, but not during LN (Figs. 4e,h). Conversely, the anomalous wave-2 activity following eastern Pacific blocks is mostly restricted to LN winters (cf. Figs. 4c and 4f,i). Note also that during LN, the wave- 2 contribution of eastern Pacific blocks to the total eddy heat flux is strong enough to overcome the negative contribution from wave 1 (Fig. $4 \mathrm{~g}$ ). This is not the case during EN, when the wave-1 effect of eastern $\mathrm{Pa}$ cific blocks is dominant (Fig. 4d). In summary, these results indicate that the mean effect of regional blocking on the polar vortex is ENSO dependent and this ENSO modulation coincides reasonably well with that found for the blocking precursors of SSWs. Thus, the ENSO modulation of the relationship between blocking and the polar vortex is quite independent on whether or not blocks lead to SSWs.

\section{c. ENSO modulation of SSWs}

We have shown that, on average, Atlantic blocks during EN and eastern Pacific blocks during LN perturb significantly the polar vortex through the amplification of wave 1 and wave 2 , respectively. In addition, these blocking regions also act as the main blocking precursors of SSWs during EN and LN, respectively (section 3a). Hence, it is reasonable to expect some association between ENSO and the wave signatures prior to SSWs, with a tendency for wave-1 signatures before EN SSWs and wave-2 features prior to LN SSWs. To explore this relationship, we computed the temporal evolution of $Z_{1}$ and $Z_{2}$ anomalies for the period from -30 to 30 days around the central date of SSWs (Fig. 6). Similar results are obtained if the composites are computed for the wave components of the poleward heat flux. The results confirm that EN SSWs are frequently preceded by wave1 amplification (Fig. 6b), whereas LN SSWs preferably occur after wave-2 amplification (Fig. 6c).

Figure 6 also shows the ENSO-based composites corresponding to each type of SSW. It is found that displacement SSWs are associated with wave-1 amplification during both EN (Fig. 6e) and LN (Fig. 6f) and some reduction in wave 2 . This is in agreement with their respective blocking precursors (European blocks during EN and Siberian blocks during LN, Figs. 2c,e), which act to enhance the upward propagation of wave 1 (Fig. 4b). However, there are major differences in the wave preconditioning of splitting SSWs between EN and LN. During LN, splitting SSWs occur after a significant wave2 amplification (Fig. 6i), in agreement with the blocking precursors of this type of SSW (eastern Pacific blocks, Fig. 2f) and their effects on wave 2 (Fig. 4i). However, splitting SSWs are associated to wave-1 amplification during EN (Fig. 6h), in accordance with the wave-1 response to Atlantic blocks (Fig. 4e), which are the main precursors of this type of SSWs during EN (Fig. 2d). Therefore, and despite the traditional belief of vortex splits being associated with wave 2 , our results indicate 
a) SSW

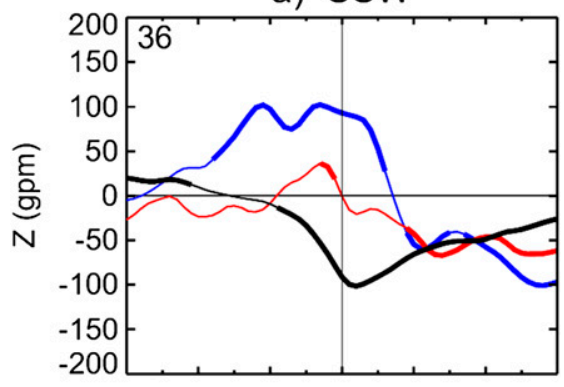

d) SSW-displ

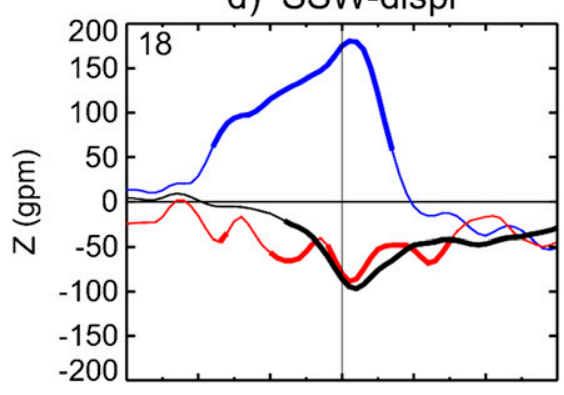

g) SSW-split

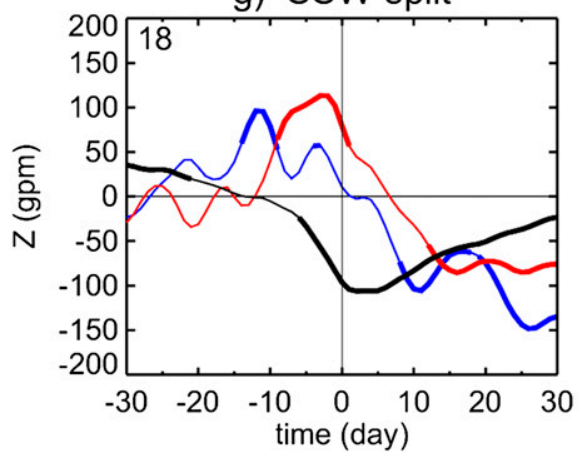

b) EN SSW

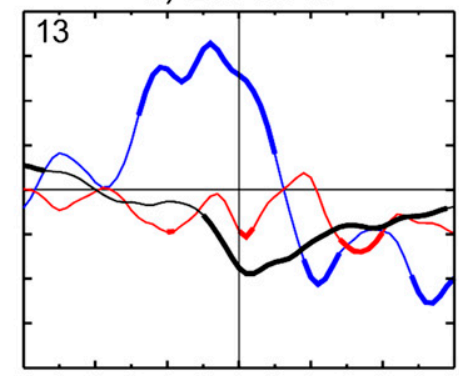

e) EN SSW-displ

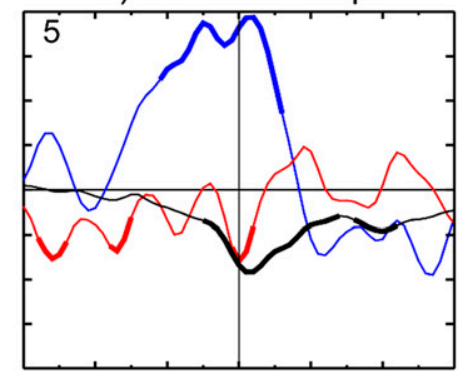

h) EN SSW-split

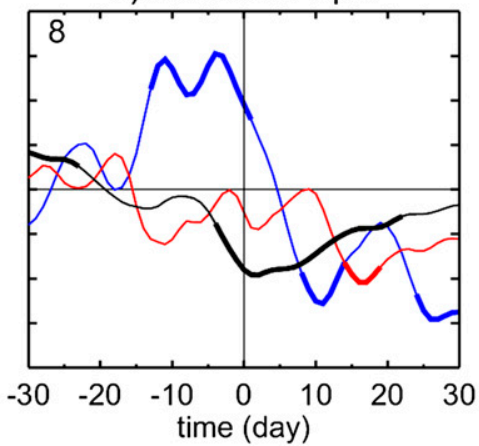

c) LN SSW

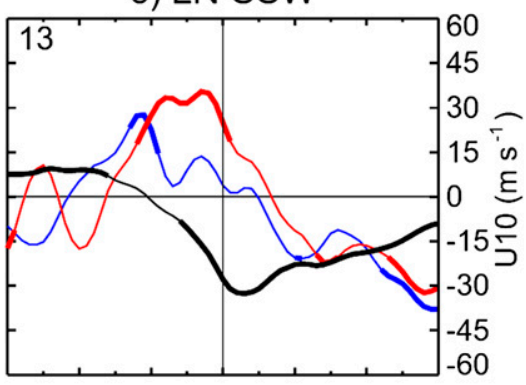

f) LN SSW-displ

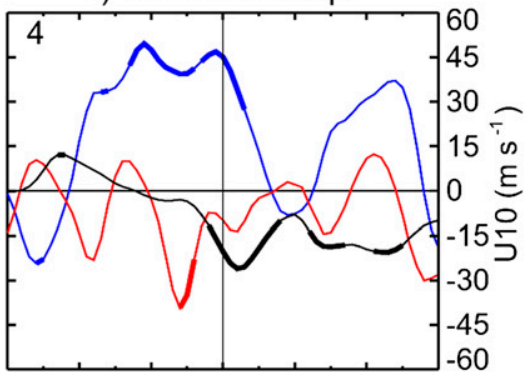

i) LN SSW-split

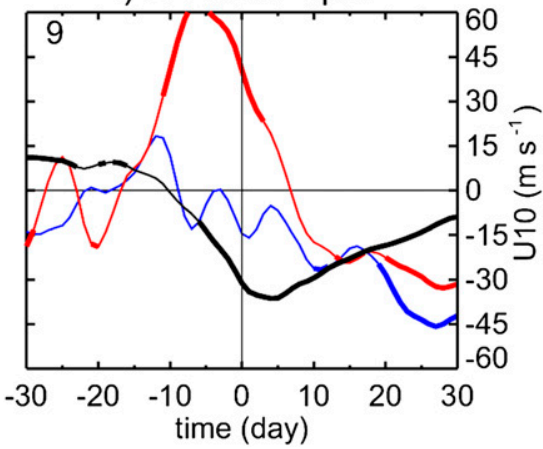

FIG. 6. Composites of the temporal evolution of 50-hPa geopotential height wave-1 ( $Z_{1}$; blue) and wave-2 $\left(Z_{2}\right.$; red) anomalies at $60^{\circ} \mathrm{N}$ $(\mathrm{gpm})$ and of the zonal mean zonal wind anomalies $\left(\mathrm{m} \mathrm{s}^{-1}\right)$ at $10 \mathrm{hPa}$ and $60^{\circ} \mathrm{N}$ (black) for the [-30,30]-day period around the central date of SSWs. The temporal evolution for (a)-(c) all SSWs, (d)-(f) displacement SSWs, and (g)-(i) splitting SSWs. The corresponding composites during (left) all, (center) EN, and (right) LN winters. The numbers in the top-left corner of each panel indicate the sample size of the composite. The periods highlighted with thickened lines indicate significant differences with respect to climatology at the $95 \%$ confidence level. The significance is assessed with a Monte Carlo test of 5000 samples with the same number of cases and calendar days as the SSWs of each composite but with random years of occurrence.

that, for the classification of SSWs adopted here, there is not a one-to-one correspondence between the occurrence of splitting SSWs and a preconditioned wave-2 forcing. This was also stressed by Bancalá et al. (2012), who found that nearly half of the splitting SSWs that occurred during the 1958-2002 period did not exhibit wave-2 signatures. They argued that this mismatch was a result of the classification criterion of SSWs into splitting and displacement events, which strongly relies on the postwarming period of the SSW rather than on the wave activity before the SSW. Our results further reveal that the lack of association between splitting SSWs and wave- 2 events is ENSO dependent, being particularly apparent during EN, but not during LN.

The absence of wave-2 signatures associated to splitting SSWs during EN is further supported by Fig. 7, which shows the composited fields of equivalent latitude at $700 \mathrm{~K}$ around the central date of SSWs. The equivalent latitude considers the area enclosed within a given $\mathrm{PV}$ contour on an isentropic surface, and provides a very useful vortex tracer (e.g., Nash et al. 1996). In contrast to the LN composite (Fig. 7f), splitting SSWs during EN 
a) EN SSW

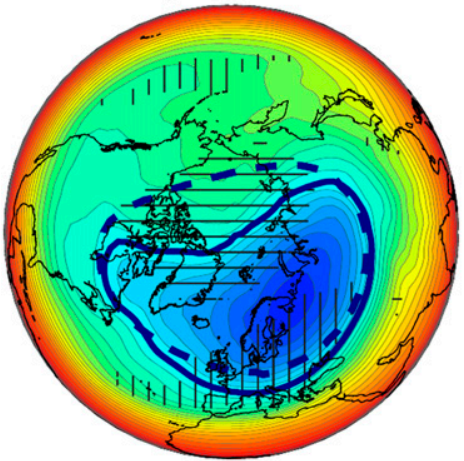

d) LN SSW

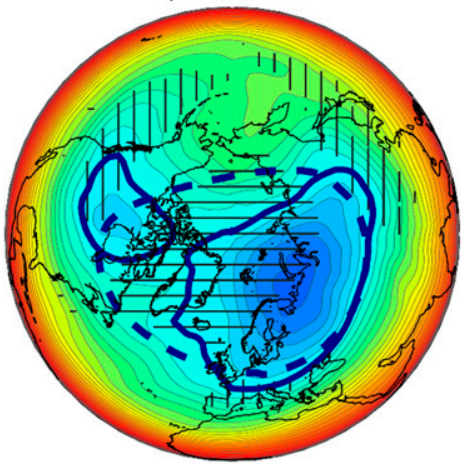

b) EN SSW-displ.

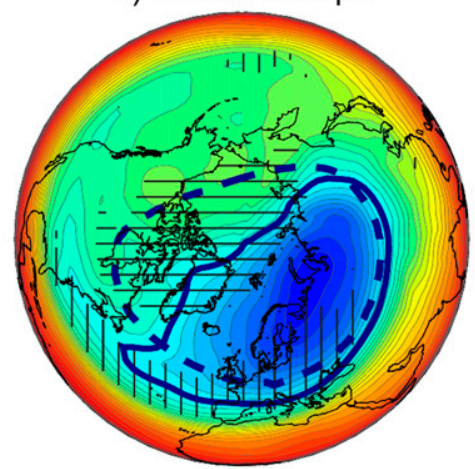

e) LN SSW-displ.

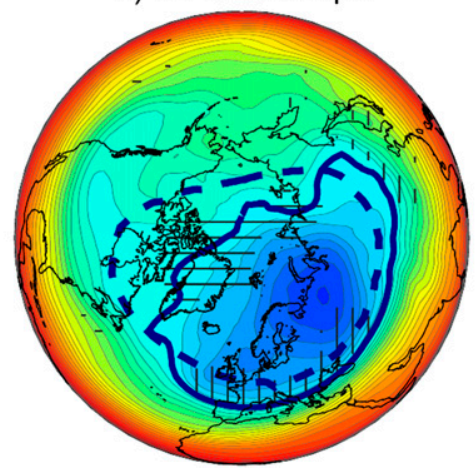

c) EN SSW-split

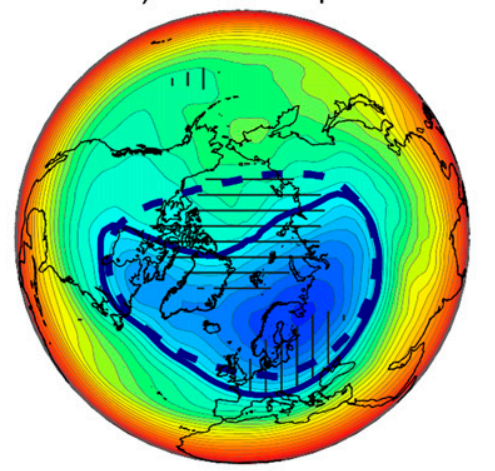

f) LN SSW-split

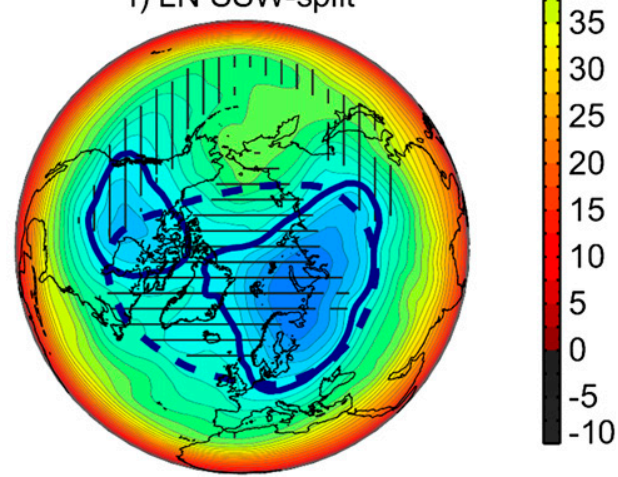

FIG. 7. Composites of equivalent latitude $\left({ }^{\circ} \mathrm{N}\right)$ at $700 \mathrm{~K}$ for the $[-5,5]$-day period of (a),(d) all SSWs; (b),(e) displacement SSWs; and (c),(f) splitting SSWs that occur during (a)-(c) EN and (d)-(f) LN winters. Blue solid (dashed) line identifies the composited (climatological) polar vortex, herein defined as the equivalent latitude of $55^{\circ} \mathrm{N}$. Above (below) normal values that are significant at $p>95 \%$ are cross hatched with vertical (horizontal) lines. The significance was assessed with a Monte Carlo test of 1000 trials in which the same number of SSW cases of each composite is chosen with random years, but keeping their days and months intact.

(Fig. 7c) do not show the typical wave-2-like pattern associated with the vortex division in two pieces, but a vortex deformation with equatorward shift and extension toward Canada that rather resembles a displacement SSW.

These results also imply that a wave- 1 forcing during EN does not necessarily mean a high probability of occurrence of a displacement SSW, given that both displacement and splitting SSWs preferentially display a wave-1 preconditioning during that ENSO phase (Figs. 6e,h). In fact, there are not significant differences between the frequency of both types of SSWs during EN (Fig. 3a). On the other hand, Figs. 6b,c do suggest a tendency for wave-1 SSWs during EN and for wave-2 SSWs during LN. This is confirmed by the frequency distribution of wave- 1 and wave-2 SSWs during different ENSO phases shown in Fig. 3b. In fact, all SSWs that occurred during EN were wave-1 events. During LN, there is not such a biased distribution toward wave-2 events, although the frequency of wave-1 (wave-2) SSWs is significantly reduced (enhanced), as compared to climatology. As a consequence, the frequency difference between wave-1 and wave-2 SSWs does reveal significant positive values during EN and negative ones during $\mathrm{LN}$ (Fig. 3b, bottom plot). The strong connection between EN and wave-1 SSWs and the preference for wave-2 SSWs during LN is in agreement with the modeling study of Taguchi and Hartmann (2006). However, and contrary to our reanalysis results, these authors also found more SSWs during EN than during LN, which may be due to differences in the definition of SSWs, the short observational record, or model deficiencies, such as the underestimation of wave 2 , as described in a more recent version of that model by de la Torre et al. (2012), or the reported problems in the representation of extratropical ENSO teleconnections (Garfinkel et al. 2012).

In short, the stratification of SSWs into wave-1 and wave- 2 events provides a clearer picture of the ENSO influence on the characteristics of the SSWs than that obtained from the SSW classification into vortex splits and displacements. As the grouping of SSWs into wave1 and wave- 2 events is based on the stratospheric wave activity before the SSWs, these results further confirm the ENSO modulation of the SSWs' precursors. In fact, 
a) EN block freq.

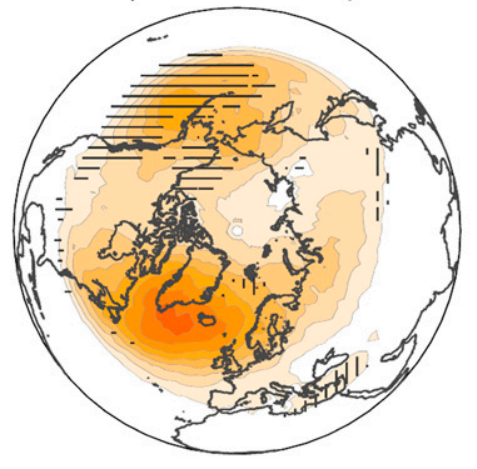

d) EN block dur.

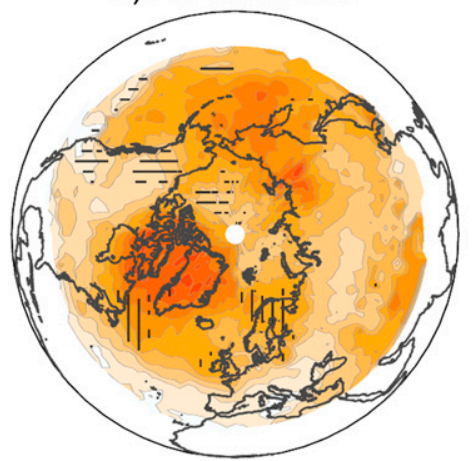

b) LN block freq.

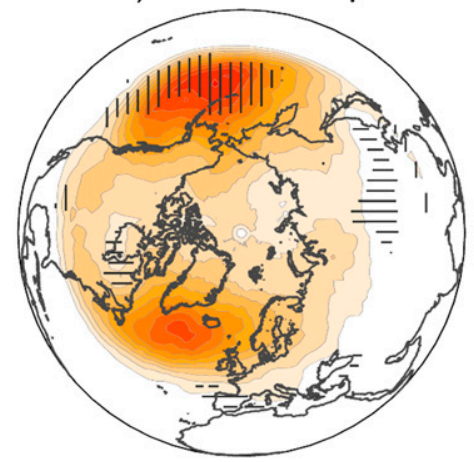

e) LN block dur.

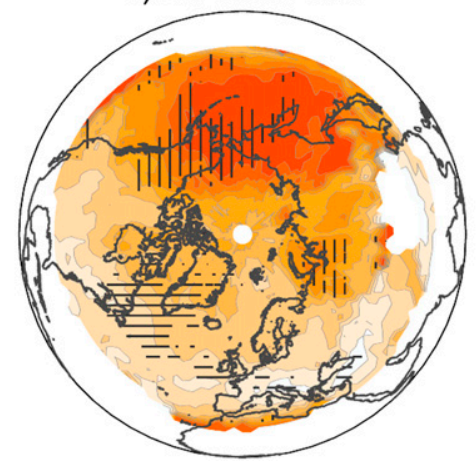

c) EN-LN block freq.

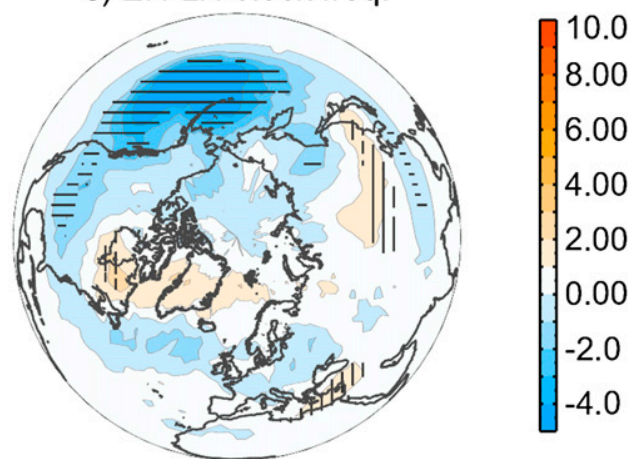

f) EN-LN block dur.

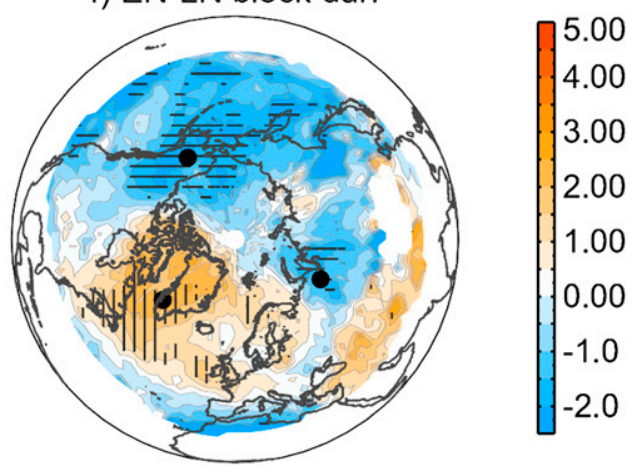

FIG. 8. Composites of winter blocking frequency (\% of winter days) for (a) EN; (b) LN, and (c) EN minus LN winters; (d)-(f) as (a)-(c), but for the mean winter blocking persistence (days). In (d) and (e) the mean blocking duration is expressed as the number of days above 5 days (the minimum blocking duration) so that a value of $d$ actually means $d+5$ days. Cross-hatched areas with vertical (horizontal) lines indicate above (below) normal values that are statistically significant at $p>95 \%$ confidence level after a Monte Carlo test of 1000 samples, each one having random years and the same size as the number of EN and LN winters. Black dots in (f) highlight selected locations.

the composite difference of the blocking precursors of SSWs between EN and LN (Fig. 1d) resembles that obtained between wave-1 and wave-2 SSWs (Fig. 1f).

\section{d. On the causes behind the ENSO influence on the blocking precursors of SSWs}

Previous studies have suggested the presence of distinguishable signatures in the tropospheric circulation anomalies that precede SSWs. For example, Nishii et al. (2011) reported that quasi-stationary blocks over the eastern Pacific region were more prone to cause pronounced changes in the stratospheric circulation, and Sjoberg and Birner (2012) found that SSWs were preferentially preceded by long-lasting tropospheric forcing, rather than by intense anomalies. In this section, we explore the possibility of ENSO influencing certain characteristics of regional blocking that, in turn, favor the occurrence of SSWs. This involves two steps: 1) identifying an ENSO signal in any of the mean characteristics of regional blocking (which should be coherent with the ENSO modulation of the blocking precursors), and
2) showing that such a change in the blocking characteristics increases the likelihood for blocking to precede a SSW. We consider blocking frequency and persistence as the features of regional blocking to be tested.

We first examine the spatial winter distribution of blocking frequency and persistence with respect to the ENSO phase (Fig. 8). These composites are computed with all winter blocking episodes, including the blocking precursors of SSWs, although we obtain similar results if the blocking precursors are removed before compositing. The null hypotheses are that blocking frequency and persistence do not change with the ENSO phase. Note that the rejection of one of the null hypotheses does not involve the rejection of the other one, since a change in blocking frequency (i.e., the number of winter blocking days) can arise by a change in the number of blocking episodes and/or in blocking persistence.

The composites of blocking frequency (Figs. 8a-c) reveal an ENSO effect on blocking frequency over the Pacific sector, with eastern Pacific blocks being significantly more frequent during LN than during EN. This is 
in agreement with the enhanced frequency of Pacific blocks prior to SSWs during LN (Fig. 1c) and its reduced activity before EN SSWs (Fig. 1b). Therefore, the preference for Pacific blocking during $\mathrm{LN}$ is not restricted to the occurrence of SSWs, but it is a climatological feature of ENSO. The ENSO modulation of eastern Pacific blocks agrees with the well-known extratropical manifestation of ENSO [i.e., the Pacific North American pattern (PNA)] and the negative correlation of the latter with Pacific blocking frequency (Croci-Maspoli et al. 2007; see also Renwick and Wallace 1996). However, LN does not increase the mean winter frequency of Siberian blocks (Fig. 8b), as it was found prior to LN SSWs. Finally, although Atlantic winter blocking tends to be more frequent during EN than during LN (Fig. 8c), similar to what was reported for the blocking precursors of SSWs (Fig. 1d), the ENSO influence on Atlantic blocking activity is not statistically significant at $p>95 \%$ (Fig. 8a). Therefore, we conclude that the ENSO modulation of blocking frequency cannot fully explain the ENSO differences in the blocking precursors of SSWs.

On the other hand, the ENSO differences in blocking persistence (Figs. 8d-f) indicate that blocks tend to be significantly longer lived over eastern Pacific and Siberia during LN and over western Atlantic during EN. Except for Europe, where the ENSO signal is weak, these are also the regions of blocking precursors of SSWs during LN and EN, respectively (Fig. 1d). In addition, the regions where the mean blocking persistence is significantly enhanced by ENSO (Fig. 8f) tend to coincide with the areas where there is an ENSO-related increase in the stratospheric poleward heat flux response to blocking (Fig. 4). The idea that blocking persistence enhances its effects in the polar stratosphere was also pointed out by Nishii et al. (2011), who argued that a block can increase the positive interference with the planetary waves by staying a long time over the stationary ridge. In summary, we find that ENSO modulates the regional mean persistence of blocking, and the spatial imprints of this ENSO signal resemble the ENSO differences in the blocking precursors of SSWs.

Thus, it remains to be shown that a longer persistence in regional blocking actually favors the occurrence of SSWs. To address this question we explored the changes in the blocking predictive skill of SSWs with respect to the blocking duration. To do so, we identified the occurrence of blocking patterns over regions lying within $500 \mathrm{~km}$ from selected grid points that are representative of the key blocking precursor regions (Nishii et al. 2011). We then grouped the blocking cases according to their persistence over the selected area (note that as the selected regions are relatively small, a high persistence of a blocking pattern therein also involves high quasi stationarity). Finally, we tested if the changes in regional blocking persistence involve an improved linkage with SSWs by simply computing the conditional probability (\%) of SSW occurrence given the occurrence of blocking:

$P_{\mathrm{SSW} \mid \text { block }}(r, d)=100 f_{\text {block } \rightarrow \mathrm{SSW}}(r, d) / f_{\text {block }}(r, d)$,

where $f_{\text {block } \rightarrow \text { SSW }}$ is the frequency of regional blocking episodes within 10 days before the SSW, and $f_{\text {block }}$ is the total number of regional blocking events (regardless whether or not they are followed by a SSW). Equation (1) is computed separately for all blocking episodes with durations equal or longer than a given value $d$, and $r$ denotes the blocking region. To account for the ENSO differences in the predictive skill of regional blocking, we display the conditional probability $P_{\mathrm{SSW} \text { block }}$ only during the ENSO phase for which the regional block is a precursor of SSWs. The distribution of this diagnostic as a function of the blocking duration is shown in Fig. 9 for the main precursor regions of SSWs (Fig. 1). As expected, the results indicate improvements in the predictability of SSWs during specific ENSO phases depending on the blocking sector, with Atlantic (Pacific and Siberian) blocks enhancing significantly the probability of SSWs occurrence during EN (LN), as compared to that expected from climatology. Moreover, it is found that a high persistence of blocking over these regions improves its precursor role of SSWs.

In summary, our results reveal that blocks tend to be longer lived over the western Atlantic during EN and over the eastern Pacific and Siberia during LN (Fig. 8f), this change in blocking persistence being a favorable condition for the occurrence of SSWs (Fig. 9). Therefore, the spatial differences in the blocking precursors of EN and LN SSWs (Fig. 1) could be explained by the ENSO modulation on the mean blocking persistence.

\section{Conclusions}

In this study we have assessed the effect of ENSO on the relationship between blocking and the polar vortex in both the full dataset and the smaller subset of SSWs, therein including the ENSO influence on the blocking precursors of SSWs and on different diagnostics of SSWs, such as the relative frequency distribution of each type of SSW and the wave preconditioning of the polar vortex. Most of these SSW signatures exhibit significant differences between opposite phases of ENSO. The main results can be summarized as follows:

1) SSWs are preceded by blocking patterns that occur over different regions depending on the ENSO phase: an enhanced activity of Atlantic blocking tends to precede SSWs during El Niño (EN), while eastern 

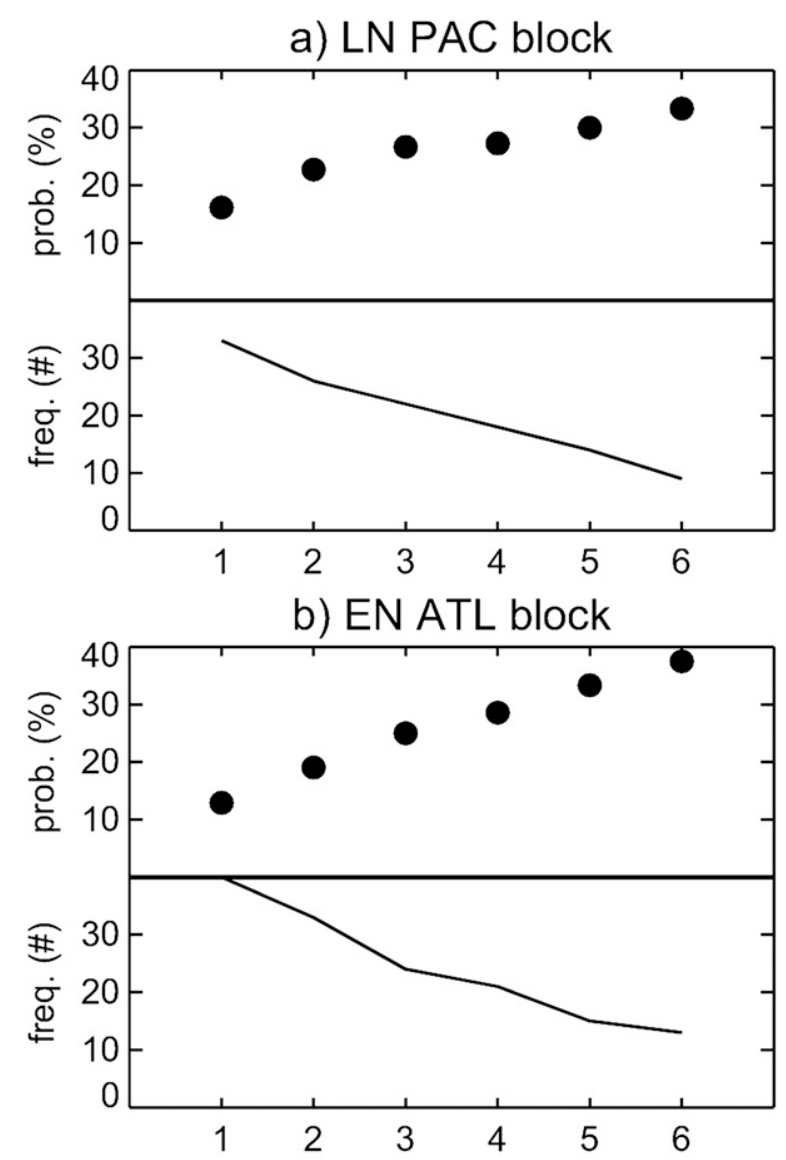

c) LN SIB block

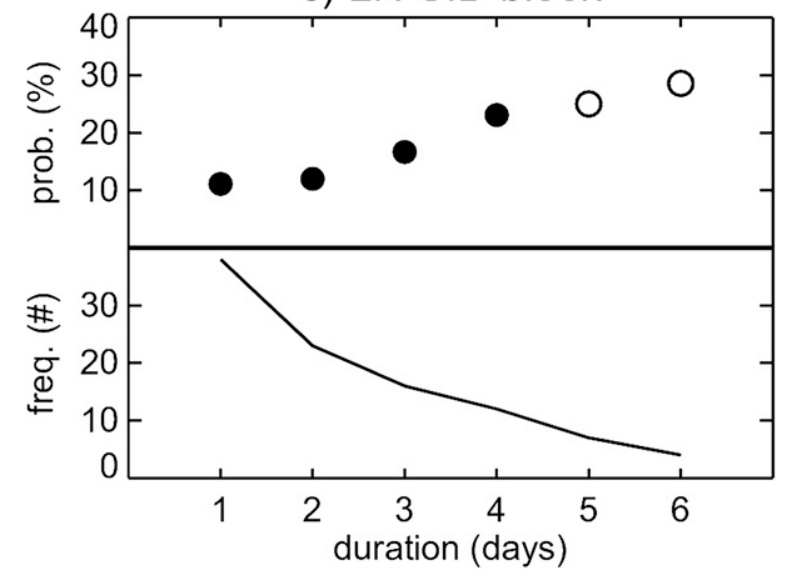

FIG. 9. Distribution of the blocking predictive skill of SSWs (\%) as a function of the time that blocking stays over regions of $\pm 500 \mathrm{~km}$ centered on selected points (highlighted in Fig. 8f), shown in the top plots for (a) Pacific $\left(65^{\circ} \mathrm{N}, 150^{\circ} \mathrm{W}\right)$ during $\mathrm{LN}$, (b) Atlantic $\left(65^{\circ} \mathrm{N}\right.$, $\left.50^{\circ} \mathrm{W}\right)$ during $\mathrm{EN}$, and (c) Siberia $\left(65^{\circ} \mathrm{N}, 65^{\circ} \mathrm{E}\right)$ during $\mathrm{LN}$. The distribution of the frequency of regional blocking episodes with duration equal or longer than the indicated value are shown in the bottom plots of (a)-(c). The skill is defined as the conditional probability of SSWs occurrence under the occurrence of a block (see text for details). Filled circles indicate that the probability is significantly higher than that expected from climatology at the $p>95 \%$ confidence level, as derived from a Monte Carlo test of 1000 samples by selecting randomly the years of EN and LN winters.
Pacific blocks are the preferred precursors of SSWs during La Niña (LN). This ENSO signal on the blocking precursors of SSWs is larger than that obtained in previous studies by stratifying the SSWs into splitting and displacement SSWs. The relatively weaker difference between the blocking predictors of splitting and displacement SSWs seems to arise from the diversity of precursors in both types of SSWs. In fact, there are also ENSO differences in the blocking precursors of splitting and displacement SSWs.

2) The blocking influence on the polar vortex is sensitive to ENSO, regardless of whether or not the blocking events yield SSWs. This spatial ENSO modulation coincides with the ENSO differences in the blocking precursors of SSWs. Blocks that occur over the Atlantic and European sectors (the precursor regions of EN SSWs) cause a larger effect on the polar stratosphere during EN than during LN due to an increased wave-1 upward propagation. During LN, eastern Pacific blocking is associated with an enhanced stratospheric heat flux by wave 2 that overcomes its wave- 1 cooling effect, thus explaining the predilection for these precursors during $\mathrm{LN}$.

3) As a consequence of points 1 and 2, there are ENSO differences in the preconditioned wave activity of SSWs. As such, SSWs that develop during EN tend to be preceded by wave- 1 amplification, regardless the type of SSW (splitting or displacement), while there is a dominance of wave 2 prior to SSWs that occur during LN. However, and contrary to EN SSWs, the wave activity before LN SSWs depends on the type of SSW: splitting SSWs tend to be preceded by wave-2 amplification, whereas displacement SSWs are triggered by wave 1 .

4) The ENSO effect on the wave signatures of the SSWs leads to an unbalanced frequency distribution of wave-1 and wave-2 SSWs between opposite ENSO phases. Wave-1 SSWs are more likely to occur during EN, while LN enhances the occurrence of wave-2 SSWs over wave- 1 events. However, the ENSO signal in the frequency of wave- 1 and wave-2 SSWs does not have a corresponding effect on the relative occurrence of displacement and splitting SSWs. This can be explained by the lack of correspondence between wave-2 and splitting SSWs during EN.

5) The blocking precursor regions coincide with the areas where ENSO significantly increases blocking persistence, supporting that there are specific signatures in the blocking precursors of SSWs, as compared to nonprecursor blocks. In fact, the development of long-lived blocks seems to increase the likelihood of SSWs occurrence. These findings allow us to identify a feasible mechanism by which ENSO modulates the 
blocking precursors of SSWs: ENSO influences regional blocking persistence, which, in turn, modulates the blocking effect on the polar stratosphere and changes the odds for blocking to trigger SSWs.

This study contributes to our understanding of the ENSO influence on SSWs, including LN winters, which for a long time were not thought to be particularly prone to the occurrence of these extreme events. Our results also involve a potential improvement in the prediction of SSWs based on the knowledge of the ENSO phase and suggest that more skillful predictions of SSWs are expected to be gained, not only from the simple occurrence of blocking, but also from its persistence.

Acknowledgments. This study was supported by the Spanish Ministry of Science and Innovation (MCINN) through the MATRES (CGL2012-34221) project, the Portuguese Science Foundation (FCT) through the ENAC (PTDC/AAC-CLI/103567/2008) project, and the EU FP7 program through the StratoClim project (Reference number 603557). We thank to R. García-Herrera and R. R. García for their comments. We want to acknowledge Olivia Martius and two additional reviewers who contributed to improvements in this manuscript.

\section{REFERENCES}

Andrews, D. G., J. R. Holton, and C. B. Leovy, 1987: Middle Atmosphere Dynamics. International Geophysics Series, Vol. 40, Academic Press, 489 pp.

Baldwin, M. P., and T. J. Dunkerton, 2001: Stratospheric harbingers of anomalous weather regimes. Science, 294, 581-584, doi:10.1126/science.1063315.

, D. B. Stephenson, D. W. J. Thompson, T. J. Dunkerton, A. J. Charlton, and A. O'Neill, 2003: Stratospheric memory and skill of extended-range weather forecasts. Science, 301, 636640, doi:10.1126/science.1087143.

Bancalá, S., K. Krüger, and M. Giorgetta, 2012: The preconditioning of major sudden stratospheric warmings. J. Geophys. Res., 117, D04101, doi:10.1029/2011JD016769.

Barriopedro, D., R. García-Herrera, and R. M. Trigo, 2010: Application of blocking diagnosis methods to General Circulation Models. Part I: A novel detection scheme. Climate Dyn., 35, 1373-1391, doi:10.1007/s00382-010-0767-5.

Butler, A. H., and L. M. Polvani, 2011: El Niño, La Niña, and stratospheric sudden warmings: A reevaluation in light of the observational record. Geophys. Res. Lett., 38, L13807, doi:10.1029/2011GL048084.

Calvo, N., R. R. García, W. J. Randel, and D. R. Marsh, 2010: Dynamical mechanism for the increase in tropical upwelling in the lowermost tropical stratosphere during warm ENSO events. J. Atmos. Sci., 67, 2331-2340, doi:10.1175/2010JAS3433.1.

Castanheira, J. M., and D. Barriopedro, 2010: Dynamical connection between tropospheric blockings and stratospheric polar vortex Geophys. Res. Lett., 37, L13809, doi:10.1029/2010GL043819.

Charlton, A. J., and L. M. Polvani, 2007: A new look at stratospheric sudden warmings. Part I: Climatology and modeling benchmarks. J. Climate, 20, 449-469, doi:10.1175/JCLI3996.1.
Cohen, J., and J. Jones, 2011: Tropospheric precursors and stratospheric warmings. J. Climate, 24, 6562-6572, doi:10.1175/ 2011JCLI4160.1.

Croci-Maspoli, M., C. Schwierz, and H. C. Davies, 2007: Atmospheric blocking: Space-time links to the NAO and PNA. Climate Dyn., 29, 713-725, doi:10.1007/s00382-007-0259-4.

Dee, D. P., and Coauthors, 2011: The ERA-Interim reanalysis: Configuration and performance of the data assimilation system. Quart. J. Roy. Meteor. Soc., 137, 553-597, doi:10.1002/qj.828.

de la Torre, L., R. R. Garcia, D. Barriopedro, and A. Chandran, 2012: Climatology and characteristics of stratospheric sudden warmings in the Whole Atmosphere Community Climate Model. J. Geophys. Res., 117, D04110, doi:10.1029/2011JD016840.

Free, M., and D. J. Seidel, 2009: Observed El Niño-Southern Oscillation temperature signal in the stratosphere. J. Geophys. Res., 114, D23108, doi:10.1029/2009JD012420.

García-Herrera, R., N. Calvo, R. R. Garcia, and M. A. Giorgetta, 2006: Propagation of ENSO temperature signals into the middle atmosphere: A comparison of two general circulation models and ERA-40 reanalysis data. J. Geophys. Res., 111, D06101, doi:10.1029/2005JD006061.

Garfinkel, C. I., D. L. Hartmann, and F. Sassi, 2010: Tropospheric precursors of anomalous Northern Hemisphere stratospheric polar vortices. J. Climate, 23, 3282-3299, doi:10.1175/ 2010JCLI3010.1.

, A. H. Butler, D. W. Waugh, M. M. Hurwitz, and L. M. Polvani, 2012: Why might stratospheric sudden warmings occur with similar frequency in El Niño and La Niña winters? J. Geophys. Res., 117, D19106, doi:10.1029/2012JD017777.

Gerber, E. P., C. Orbe, and L. M. Polvani, 2009: Stratospheric influence on the tropospheric circulation revealed by idealized ensemble forecasts. Geophys. Res. Lett., 36, L24801, doi:10.1029/ 2009GL040913.

Gómez-Escolar, M., S. Fueglistaler, N. Calvo, and D. Barriopedro, 2012: Changes in polar stratospheric temperature climatology in relation to stratospheric sudden warming occurrence. Geophys. Res. Lett., 39, L22802, doi:10.1029/2012GL053632.

Holton, J. R., and H.-C. Tan, 1980: The influence of the equatorial quasi-biennial oscillation on the global circulation at $50 \mathrm{mb}$. J. Atmos. Sci., 37, 2200-2208, doi:10.1175/ 1520-0469(1980)037<2200:TIOTEQ > 2.0.CO;2.

Kalnay, E., and Coauthors, 1996: The NCEP/NCAR 40-Year Reanalysis Project. Bull. Amer. Meteor. Soc., 77, 437-471, doi:10.1175/1520-0477(1996)077<0437:TNYRP $>2.0 . C O ; 2$.

Limpasuvan, V., D. W. J. Thompson, and D. L. Hartmann, 2004: The life cycle of the Northern Hemisphere sudden stratospheric warmings. J. Climate, 17, 2584-2596, doi:10.1175/ 1520-0442(2004)017<2584:TLCOTN>2.0.CO;2.

Manzini, E., M. A. Giorgetta, M. Esch, L. Kornblueh, and E. Roeckner, 2006: The influence of sea surface temperatures on the northern winter stratosphere: Ensemble simulations with the MAECHAM5 model. J. Climate, 19, 3863-3881, doi:10.1175/JCLI3826.1.

Marshall, A. G., and A. A. Scaife, 2010: Improved predictability of stratospheric sudden warming events in an atmospheric general circulation model with enhanced stratospheric resolution. J. Geophys. Res., 115, D16114, doi:10.1029/2009JD012643.

Martius, O., L. M. Polvani, and H. C. Davies, 2009: Blocking precursors to stratospheric sudden warming events. Geophys. Res. Lett., 36, L14806, doi:10.1029/2009GL038776.

Matsuno, T., 1971: A dynamical model of the stratospheric sudden warming. J. Atmos. Sci., 28, 1479-1494, doi:10.1175/ 1520-0469(1971)028<1479:ADMOTS>2.0.CO;2. 
Matthes, K., Y. Kuroda, K. Kodera, and U. Langematz, 2006: Transfer of the solar signal from the stratosphere to the troposphere: Northern winter. J. Geophys. Res., 111, D06108, doi:10.1029/2005JD006283.

Mitchell, D. M., L. J. Gray, J. Anstey, M. P. Baldwin, and A. J. Charlton-Perez, 2013: The influence of stratospheric vortex displacements and splits on surface climate. J. Climate, 26, 2668-2682, doi:10.1175/JCLI-D-12-00030.1.

Nash, E. R., P. A. Newman, J. E. Rosenfield, and M. R. Schoeberl, 1996: An objective determination of the polar vortex using Ertel's potential vorticity. J. Geophys. Res., 101, 9471-9478, doi:10.1029/96JD00066.

Newman, P. A., and E. R. Nash, 2000: Quantifying the wave driving of the stratosphere. J. Geophys. Res., 105, 12485-12497, doi:10.1029/1999JD901191.

Nishii, K., H. Nakamura, and Y. J. Orsolini, 2011: Geographical dependence observed in blocking high influence on the stratospheric variability through enhancement and suppression of upward planetary-wave propagation. J. Climate, 24, 6408-6423, doi:10.1175/JCLI-D-10-05021.1.

Polvani, L. M., and D. W. Waugh, 2004: Upward wave activity flux as a precursor to extreme stratospheric events and subsequent anomalous surface weather regimes. J. Climate, 17, 3548-3554, doi:10.1175/1520-0442(2004)017<3548:UWAFAA > 2.0.CO;2.

Quiroz, R. S., 1986: The association of stratospheric warmings with tropospheric blocking. J. Geophys. Res., 91, 5277-5285, doi:10.1029/JD091iD04p05277.
Renwick, J. A., and J. M. Wallace, 1996: Relationships between North Pacific wintertime blocking, El Niño, and the PNA pattern. Mon. Wea. Rev., 124, 2071-2076, doi:10.1175/ 1520-0493(1996)124<2071:RBNPWB > 2.0.CO;2.

Robock, A., and J. Mao, 1992: Winter warming from large volcanic eruptions. Geophys. Res. Lett., 19, 2405-2408, doi:10.1029/ 92GL02627.

Sassi, F., D. Kinnison, B. A. Boville, R. R. Garcia, and R. Roble, 2004: Effect of El Niño-Southern Oscillation on the dynamical, thermal, and chemical structure of the middle atmosphere. J. Geophys. Res., 109, D17108, doi:10.1029/2003JD004434.

Sjoberg, J. P., and T. Birner, 2012: Transient tropospheric forcing of sudden stratospheric warmings. J. Atmos. Sci., 69, 34203432, doi:10.1175/JAS-D-11-0195.1.

Taguchi, M., 2008: Is there a statistical connection between stratospheric sudden warming and tropospheric blocking events? J. Atmos. Sci., 65, 1442-1454, doi:10.1175/ 2007JAS2363.1.

— spheric sudden warmings during El Niño simulated by WACCM. J. Climate, 19, 324-332, doi:10.1175/JCLI3655.1.

Uppala, M., and Coauthors, 2005: The ERA-40 re-analysis. Quart. J. Roy. Meteor. Soc., 131, 2961-3012, doi:10.1256/qj.04.176.

Woollings, T., A. Charlton-Perez, S. Ineson, A. G. Marshall, and G. Masato, 2010: Associations between stratospheric variability and tropospheric blocking. J. Geophys. Res., 115, D06108, doi:10.1029/2009JD012742. 
Copyright of Journal of Climate is the property of American Meteorological Society and its content may not be copied or emailed to multiple sites or posted to a listserv without the copyright holder's express written permission. However, users may print, download, or email articles for individual use. 